\title{
Las aves en la Región del Biobío (Chile): su riqueza, composición y distribución
}

\author{
Birds in the Biobío Region (Chile): their richness, composition, and distribution
}

\author{
Patricio Pellet ${ }^{1, *} \&$ Cristián Cornejo ${ }^{2}$ \\ 'BioMA, Biodiversidad y Medio Ambiente, bioma.cl, San Pedro de la Paz, Chile. \\ ${ }^{2}$ Ministerio del Medio Ambiente, Región del Biobío, Chile. \\ *E-mail: ppellet@bioma.cl
}

\section{RESUMEN}

La Región del Biobío al formar parte del "hotspot" de biodiversidad "Chilean Winter rainfall - Valdivian forests" se caracteriza por tener una tasa alta tanto de endemismo como de pérdida de hábitats y fragmentación. Aunque las aves permiten monitorear estas realidades, además de los efectos que sobre sus hábitats tiene el Cambio Climático Global (CCG), su conocimiento aún es escaso tanto en la Región del Biobío como en Chile. Un inventario regional sería costoso en tiempo, presupuesto y personal. Evaluamos la representatividad y completitud de un inventario regional obtenido a partir de registros de aves de ebird-Chile. Calculamos la riqueza específica observada en 226 especies y la riqueza esperada en 249 especies para la región. Para ello, usamos el modelo paramétrico de Clench y los modelos no paramétricos: Chao1, iChao1, ACE, Jackknife de primer orden y Jackknife de segundo orden. Identificamos 37 especies amenazadas, 6 especies endémicas y 3 especies raras. Encontramos una distribución sesgada de los esfuerzos de muestreo y diversidad de especies a comunas costeras. Una mayor atención y esfuerzo debe ponerse en el estudio y conservación de la riqueza de los taxa superiores de aves, porque representan una mayor diversidad filogenética. Estudios sobre aves en la Región del Biobío pueden usar fuentes de información como eBird-Chile para acortar la brecha de conocimiento sobre las aves, y la información obtenida puede expresarse en términos que puedan ser integrados en el ordenamiento territorial para avanzar hacia una gestión y conservación de las aves.

Palabras clave: aves, biodiversidad, conservación, ordenamiento territorial, riqueza de especies.

\section{ABSTRACT}

The Biobío Region is part of the "Chilean winter rainfall-Valdivian forests" biodiversity "hotspot" and is characterized by having a high rate of both endemism and habitat loss and fragmentation. Although birds make it possible to monitor these realities, in addition to the effects that Global Climate Change (GCC) has on their habitats, their knowledge is still scarce both in the Biobío Region and in Chile. A regional inventory would be costly on time, budget, and personnel. We evaluated the representativeness and completeness of a regional inventory obtained from bird records of ebird-Chile. The observed specific richness was 226 species and the expected richness was 249 species for the region. We used the Clench parametric model and the non-parametric models: Chao1, iChao1, ACE, First Order Jackknife, and Second-Order Jackknife for all estimations. We identified 37 threatened species, 6 endemic species, and 3 rare species. We found a biased distribution of sampling efforts and species diversity to coastal communities. Greater attention and effort should be put into the study and conservation of the richness of the higher taxa of 
birds because they represent greater phylogenetic diversity. Studies on birds in the Biobío Region can use sources of information such as eBird-Chile to bridge the knowledge gap on birds, and the information obtained can be expressed in terms that can be integrated into land-use planning to advance towards the management and conservation of birds.

Keywords: biodiversity, birds, conservation, land-use planning, species richness.

\section{INTRODUCCIÓN}

La Región del Biobío forma parte de uno de los 35 puntos críticos de diversidad o "hotspots" mundiales de biodiversidad (Myers et al. 2000). Esta área posee una superficie de 641.913 $\mathrm{km}^{2}$ (Habel et al. 2019), aloja un número de plantas endémicas que alcanza a 1.957 especies, posee un área protegida de $130.866 \mathrm{~km}^{2}$ (20,39\%), y una vegetación remanente en el "hotspot" que alcanza a un $34,2 \%$ de su superficie. Además, existen alrededor de 226 especies de aves, 43 especies de anfibios (29 endémicas, i.e., 67\%), 41 especies de reptiles (27 endémicas, i.e., 66\%), y 64 especies de mamíferos (13 endémicas, i.e., 20\%; Arroyo et al. 2006). En Chile, el número de aves endémicas alcanza las 11 especies (Barros et al. 2015, Medrano et al. 2018). Hay que mencionar, además, que de acuerdo con Stotz et al. (1996), existen 22 especies endémicas compartidas entre Chile y Argentina, a través de la cordillera de los Andes. Esto, representa una tasa de endemismo de 2,2\% (Barros et al. 2015), considerada baja en comparación con otros grupos faunísticos, como peces dulceacuícolas, reptiles y anfibios, que presentan endemismos altos (> 50\%) (Carrasco-Lagos et al. 2012). A pesar de ello, en Chile el grupo de vertebrados de mayor riqueza específica es el de las aves, dentro de las cuales, aproximadamente un $29 \%$ pertenece a los ambientes acuáticos (Victoriano et al. 2006). En este y los otros "hotspots" concentraciones excepcionales de especies endémicas están experimentando una importante pérdida de hábitat (Myers et al. 2000), siendo la consecuencia más importante la pérdida de biodiversidad, fenómeno que no es al azar, sino que sigue patrones y procesos bien definidos que se encuentran asociados, principalmente, con la actividad humana y con las zonas donde se distribuye y concentra la biodiversidad (Meffe \& Carroll 1994).

La pérdida de los hábitats y su fragmentación, son facilitados, controlados y aún incentivados por mecanismos cuyo origen se encuentra tanto en el ordenamiento territorial como en la legislación ambiental. Esto resulta paradójico si se considera que, en principio, ambos buscan establecer un control más o menos racional del uso y conservación del sistema natural. Por lo mismo, en la actualidad, se ha propuesto que este desarrollo se produzca sobre la base de objetivos de sustentabilidad para la Región del Biobío ( $\mathrm{BIOBIO}$ - Estrategia Regional de Desarrollo Periodo 20152030». Subdere, s.f.). Esto implica integrar los diferentes usos del suelo (que representan diferentes intereses en el territorio), en un escenario que asegure la satisfacción de nuestras necesidades y las de futuras generaciones, cuestión que no podrá alcanzarse si no se considera al sistema natural, donde las aves son un elemento relevante. Cabe destacar que los espacios no urbanos (e.g., bosques, humedales, praderas y matorrales, plantaciones forestales, terrenos agrícolas) ocupan un $98,9 \%$ de la superficie total regional (CONAF 2019), sus límites con lo urbano son cada vez más difusos, y no han sido incorporados en esquemas de planificación integral (Berdegué et al. 2010), a pesar de que en ellos se encuentra la casi totalidad de los ecosistemas en que se sustenta la biodiversidad de la Región del Biobío. A lo anterior, se suma otro importante factor de transformación de los hábitats de las aves, el cambio climático global (CCG), que provoca la modificación de los patrones de precipitación y temperatura de los lugares que ocupan las especies, influyendo también en la distribución, la abundancia y la relación de las especies con el ambiente (Botero 2015; Feria-Arroyo et al. 2013). Las aves permiten monitorear estos efectos del CCG en ecosistemas naturales o con influencia humana (Feria-Arroyo et al. 2013). Por lo que, conocer su número y distribución espacial es absolutamente necesario para el diseño de políticas efectivas de conservación de la biodiversidad (Hortal \& Lobo 2002). Esperar la recolección de los datos necesarios, a escala regional, podría significar una inacción que destruiría el esfuerzo de conservación (Meffe \& Carroll 1997). Ante este desafío, es claro que se requiere tomar acción sin un conocimiento completo.

En la Región del Biobío, la investigación científica sobre aves se ha centrado principalmente en las Áreas Silvestres Protegidas del Estado (SNASPE) y en algunos sitios con evidente riqueza avifaunística (Jiménez 1999; Carrasco Lagos 2004; Figueroa et al. 2000; González et al. 2011; Riffo 
\& Villarroel 2000; Riquelme et al. 2018), a pesar de ello, aún queda una gran extensión del territorio sin estudiar y, en general, tanto en la Región del Biobío como en Chile, el conocimiento sobre las aves aún es escaso para la mayoría de las especies (Tejeda \& Medrano 2018). Una nueva alternativa para avanzar en la obtención de antecedentes relevantes para el estudio de las aves son las plataformas de ciencia ciudadana (eBird, iNaturalist, OBIS), sin embargo, en Chile, hasta ahora su uso ha sido limitado (Tejeda \& Medrano 2018), aun cuando los escasos intentos efectuados han sido de gran impacto, por ejemplo, ayudaron a generar el primer Atlas de las Aves Nidificantes de Chile (Medrano et al. 2018) y la Lista de las aves de Chile 2014 (Barros et al. 2015). Considerando el importante sesgo en los estudios de diversidad de aves a nivel regional y el amplio uso de la plataforma eBird-Chile, en este trabajo buscamos describir la diversidad de las aves presentes en la Región del Biobío utilizando como fuente de información esta plataforma de ciencia ciudadana. Para ello, se propone (a) cuantificar la riqueza específica de las aves presentes en la Región del Biobío, (b) describir su composición y distribución, y (c) relacionar estos resultados con el ordenamiento del territorio a nivel regional y comunal.

\section{MÉTODOS}

\section{ÁreA DE ESTUDIO}

El área de estudio corresponde a la Región del Biobío (Chile) que, ubicándose en el centro geográfico del país, aproximadamente, entre las coordenadas $36^{\circ} 30^{\prime} / 74^{\circ} 00$ $38^{\circ} 30^{\prime} / 71^{\circ} 00^{\prime}$, marca el inicio de la zona higromórfica que se extiende desde el río Laja hasta el extremo sur del país (Fuenzalida \& Pisano 1965). Ocupa una superficie aproximada de 2.398 .996 ha $\left(23.989,96 \mathrm{~km}^{2}\right)$, lo que representa el $1,2 \%$ de la superficie conjunta del territorio de Chile, sin contar el mar territorial (Biblioteca del Congreso Nacional s.f.) (Fig. 1).

\section{INVENTARIO}

La generación de la base de datos se basó en los registros disponibles en eBird-Chile (eBird, 2019) sobre las aves observadas en la Región del Biobío. Se depuró los registros, revisando, corrigiendo, eliminando duplicados o mal informados (nombre científico erróneo, mal escrito o inexistente), y actualizando nombres científicos sinónimos (Hortal \& Lobo 2002). Para ello, se utilizó el "software" de distribución libre R version 3.6.2 (2019-12-12) (R Core Team 2019) y RStudio version 1.2.5019 (RStudio Team 2018).

\section{ANÁLISIS DE LOS DATOS}

La representatividad del inventario se evaluó construyendo una curva de acumulación de especies basada en el número de registros de la base de datos (Hortal \& Lobo 2002; Jiménez-Valverde \& Hortal 2003; Lobo \& Martin-Piera 2002), mediante la función specaccum (método "random" con 100 permutaciones) del paquete vegan version 2.5-6 (Oksanen et al. 2019), en $R$ ( $R$ Core Team 2019). Luego, siguiendo a (Jiménez-Valverde \& Hortal 2003), se ajustó el modelo asintótico de Clench, usando el método de estimación no lineal Simplex and Quasi Newton con el programa Statistica (StatSoft Inc. 2007). Se consideró un buen ajuste si $R^{2}>0,95$ (Moreno \& Halffter 2000). La ecuación de Clench tiene la forma: $S(n)=a^{*} n /\left(1+b^{*} n\right)$, donde $S(n)=$ número de especies estimadas en $\mathrm{n}, \mathrm{a}=$ tasa de incremento de nuevas especies al inicio del inventario, $b=$ acumulación de especies, $y, n=$ número acumulativo de muestras (Halffter et al. 2001; Moreno \& Halffter 2000). Este modelo, que se recomienda para estudios en áreas extensas (Jiménez-Valverde \& Hortal 2003) y cuando la intensidad de los muestreos cambia en el tiempo (Villarreal et al. 2006), plantea que la probabilidad de agregar nuevas especies aumenta (hasta un máximo) a medida que se pasa más tiempo en el campo y se adquiere mayor experiencia con el sitio, taxa y los métodos empleados (Soberón \& Llorente 1993), aun cuando la probabilidad de añadir nuevas especies eventualmente disminuye a medida que se alcanza la asíntota (Moreno 2001).

Para evaluar la completitud del inventario (C), se utilizaron dos métodos: (a) se calculó la pendiente al final de la curva ajustada. Siendo la pendiente en un punto $n=a /(1+b \cdot n)^{2} y$ usando como unidad de esfuerzo los registros de la base de datos, si ésta es menor que 0,1 nos indica que hemos logrado un buen inventario, completo y fiable (Jiménez-Valverde \& Hortal 2003); y, (b) se calculó la proporción de fauna registrada (Sobs/Sesp). Según Jiménez-Valverde \& Hortal (2003) a partir de proporciones superiores al $70 \%$ las estimaciones de la riqueza asintótica se hacen estables; otros autores proponen 85\% (Villarreal et al. 2006) y 90\% (López-Mejía et al. 2017; Moreno \& Halffter 2000).

\section{EstiMACIÓN DE LA RIQUEZA ESPECÍFICA (S)}

La riqueza específica observada (Sobs) se obtuvo del análisis sobre el inventario, y la predicción del número de especies esperadas (Sesp), siguiendo lo indicado por Villarreal et al. (2006), respecto a revisar la tendencia de varios estimadores para comparar los datos estimados con los valores observados. Se obtuvo empleando dos procedimientos: (a) usando la asíntota $(\mathrm{a} / \mathrm{b})$ de la curva de acumulación de especies (Jiménez-Valverde \& Hortal 2003; Moreno \& Halffter 2000); y, (b) empleando los estimadores no paramétricos (Moreno 2001; Villarreal et al. 2006): Chao1, iChao1, ACE, Jackknife de primer orden y Jackknife de segundo orden, a través de 
la función ChaoSpecies del paquete SpadeR version 0.1.1. (Chao et al. 2016), en R (R Core Team 2019).

La composición de especies se obtuvo generando un listado a partir de la base de datos que incluyó: Orden, Familia, Género, nombre científico, nombre común, estado de conservación y endemismo para cada una de las especies. Para ello, se usó R version 3.6.2 (2019-12-12) (R Core Team 2019) y RStudio version 1.2.5019 (RStudio Team 2018). El grado de endemismo y el estado de conservación se obtuvieron de la Nómina De Especies Según Estado Conservación Chile actualizado 15vo Proceso RCE (Ministerio de Medio Ambiente 2020).
Los mapas y gráficos son un lenguaje común en la biología de la conservación y el ordenamiento territorial, ya que se trata de elementos que pueden ser integrados en los análisis de ambas disciplinas. Por ello, la conexión de los resultados de diversidad (riqueza específica) y composición de especies con el ordenamiento territorial, se realizó elaborando mapas y gráficos para representar: a) los registros de aves por comuna, b) el número de especies por comuna, c) las especies endémicas y d) las especies amenazadas. Para ello, se usó el sistema de información geográfica QGis versión 3.12.3-București (QGIS Development Team 2015).

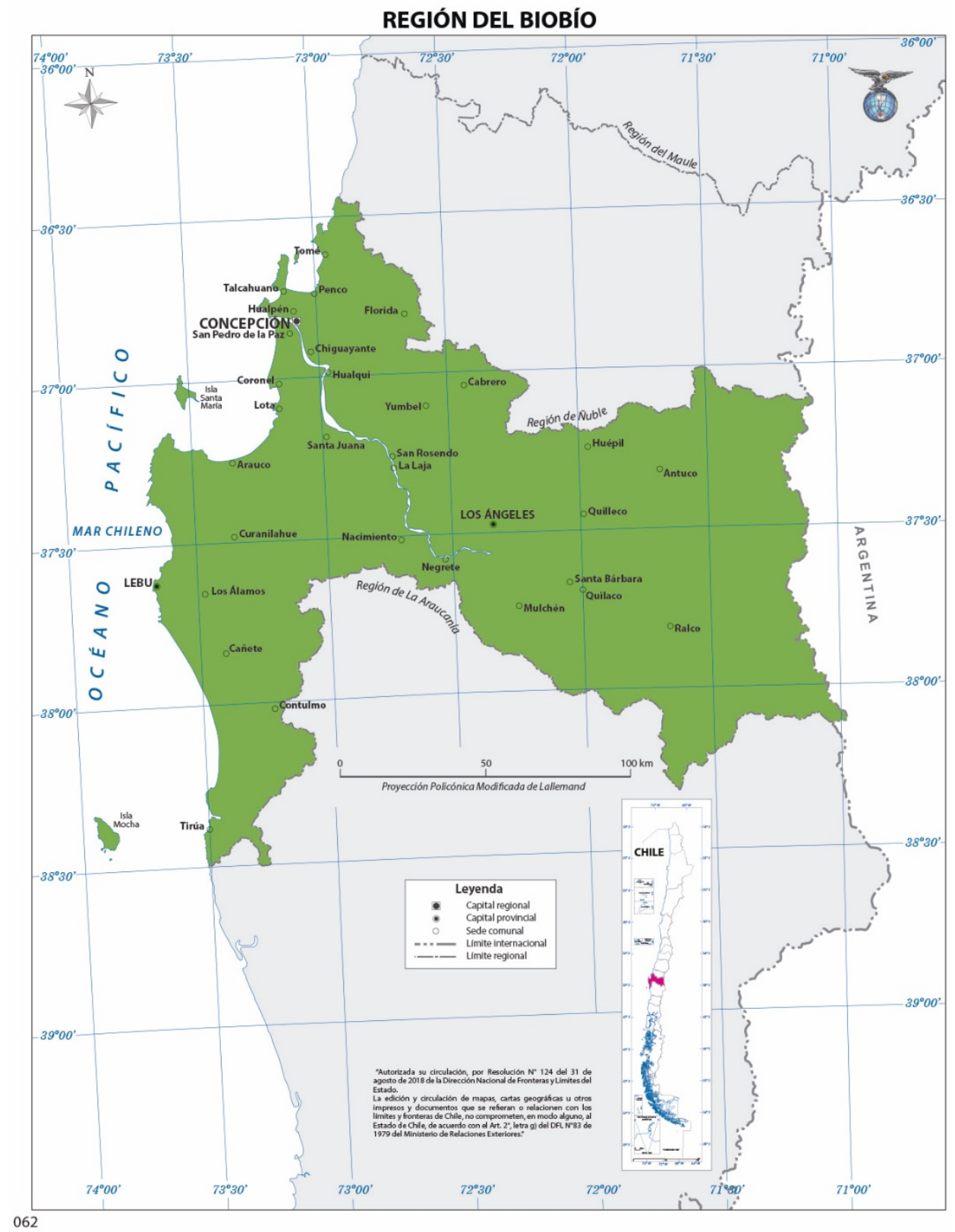

Figura 1. Área de estudio Región del Biobío (Chile). / Study area Biobío Region (Chile). 


\section{RESULTADOS}

\section{INVENTARIO}

El inventario alcanzó un total de 61.940 registros (Fig. 2 ), donde se incluye una lista completa de las especies observadas, según taxonomía de Clements et al. (2019), la latitud y longitud de las observaciones, sus fechas de registro, que abarcan desde el año 1972 hasta el año 2019 (n=47 años), y, las localidades de registro, entre otras variables.

\section{REPRESENTATIVIDAD Y COMPLETITUD DEL INVENTARIO}

La curva de acumulación de especies construida con el modelo de Clench (Fig. 3) produjo un buen ajuste para los datos, alcanzando un $\mathrm{R}^{2}=0,96(>0,95)$. Estimó un número de especies mayor que el observado ( 249 y 226 , respectivamente) con parámetros $a=0,249$ y $b=0,001$, logrando una eficiencia de muestreo de un 91\%. La completitud del inventario (C), siguiendo el método del cálculo de la pendiente al final de la curva, fue de 0,004. Mientras que la proporción de fauna registrada fue de $91 \%$, valor que es mayor a los criterios de $70 \%, 85 \%$ y $90 \%$ propuesto por varios autores (Moreno \& Halffter 2000; Jiménez-Valverde \& Hortal 2003; Villarreal et al. 2006; López-Mejía et al. 2017).

\section{ReGistros de AVES A NIVEL REGIONAL Y POR COMUNA}

En la Fig. 4 se observa que los registros a nivel regional no están bien distribuidos. Se puede apreciar que existe una mayor concentración de registros asociados al Gran Concepción, al borde costero, a la comuna de Los Ángeles, y, también, a caminos y cuerpos de agua. En cuanto al número de registros de aves por comuna, éstos se distribuyen irregularmente (Fig. 4 y Fig. 5). Existen comunas donde hay un número alto de registros, por ejemplo, Concepción y Talcahuano tienen 15.767 y 11.724 registros, respectivamente; mientras que, otras cuentan con números muy bajos, por ejemplo, San Rosendo y Nacimiento poseen 5 y 9 registros, respectivamente. En general, destacan las comunas costeras porque presentan un mayor número de registros, comparadas con comunas interiores o cordilleranas que, a su vez, destacan por presentar una baja cantidad de registros (Fig. 5). El número mínimo de registros para obtener una proporción del 85\% (Villarreal et al. 2006) del total de la avifauna registrada en la región del Biobío es de 5.667 registros. Sólo tres comunas logran este valor: Concepción, Talcahuano y Hualpén (Fig. 5).
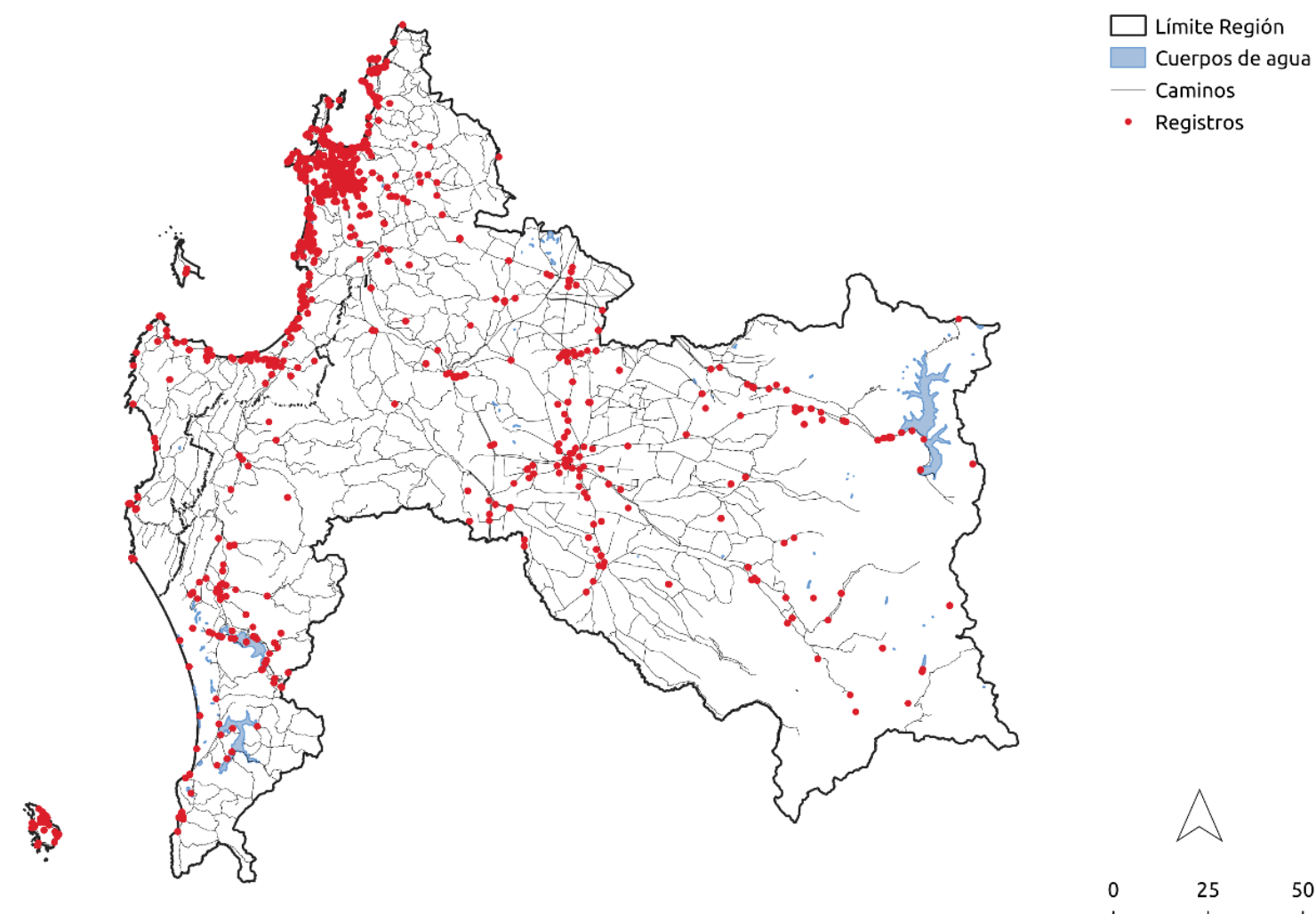

- Registros

Figura 2. Distribución de los registros que conforman la base de datos. / Distribution of the records that make up the database. 


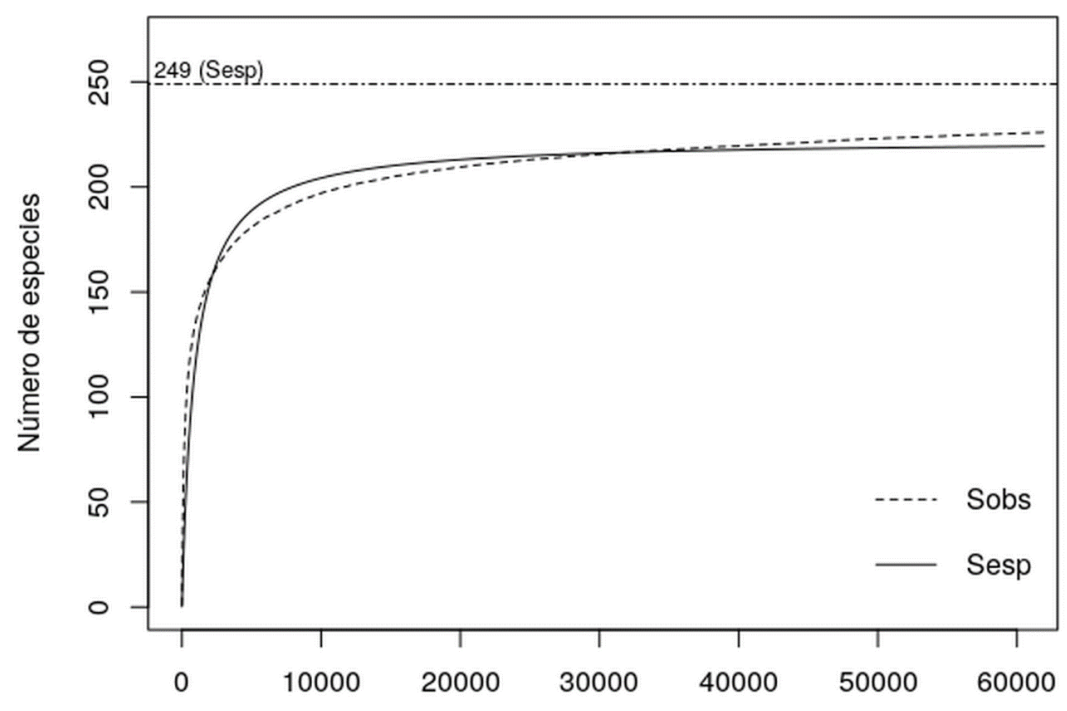

Número de registros acumulados

Figura 3. Curvas de acumulación de especies observadas (Sobs) y esperadas (Sesp, según modelo de Clench). La asíntota de la curva corresponde al número total de especies esperadas para la Región del Biobío, i.e., 249 especies. / Observed species (Sobs) and expected species (Sesp, according to Clench model) accumulation curves. The asymptote of the curve corresponds to the total number of species expected for the Biobío Region, i.e., 249 species.

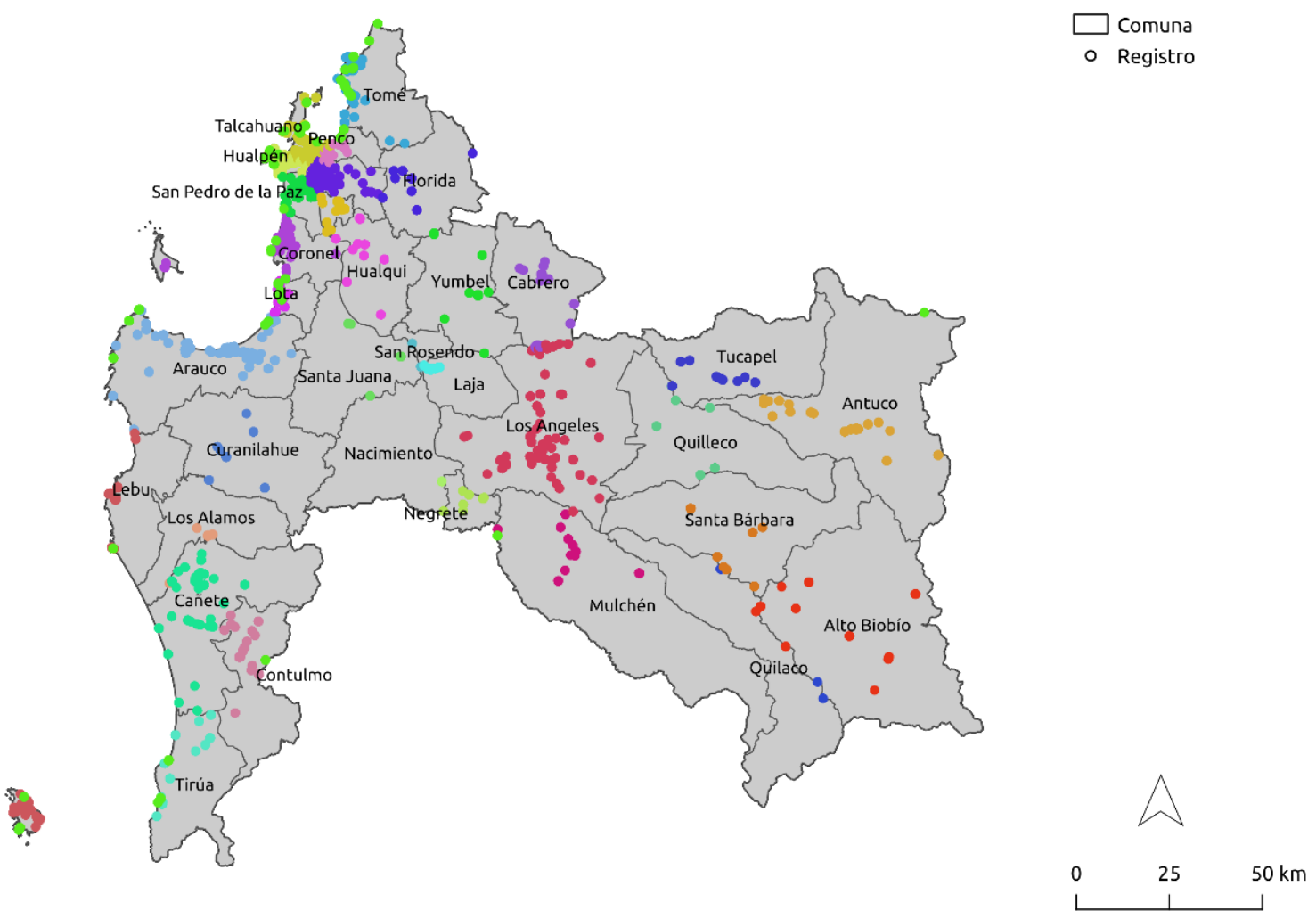

Figura 4. Distribución de los registros de aves en la Región del Biobío y por comuna. Los colores de los puntos permiten diferenciarlos por comuna. / Distribution of bird records in the Biobío Region and by commune. The colors of the points allow to differentiate them by commune. 


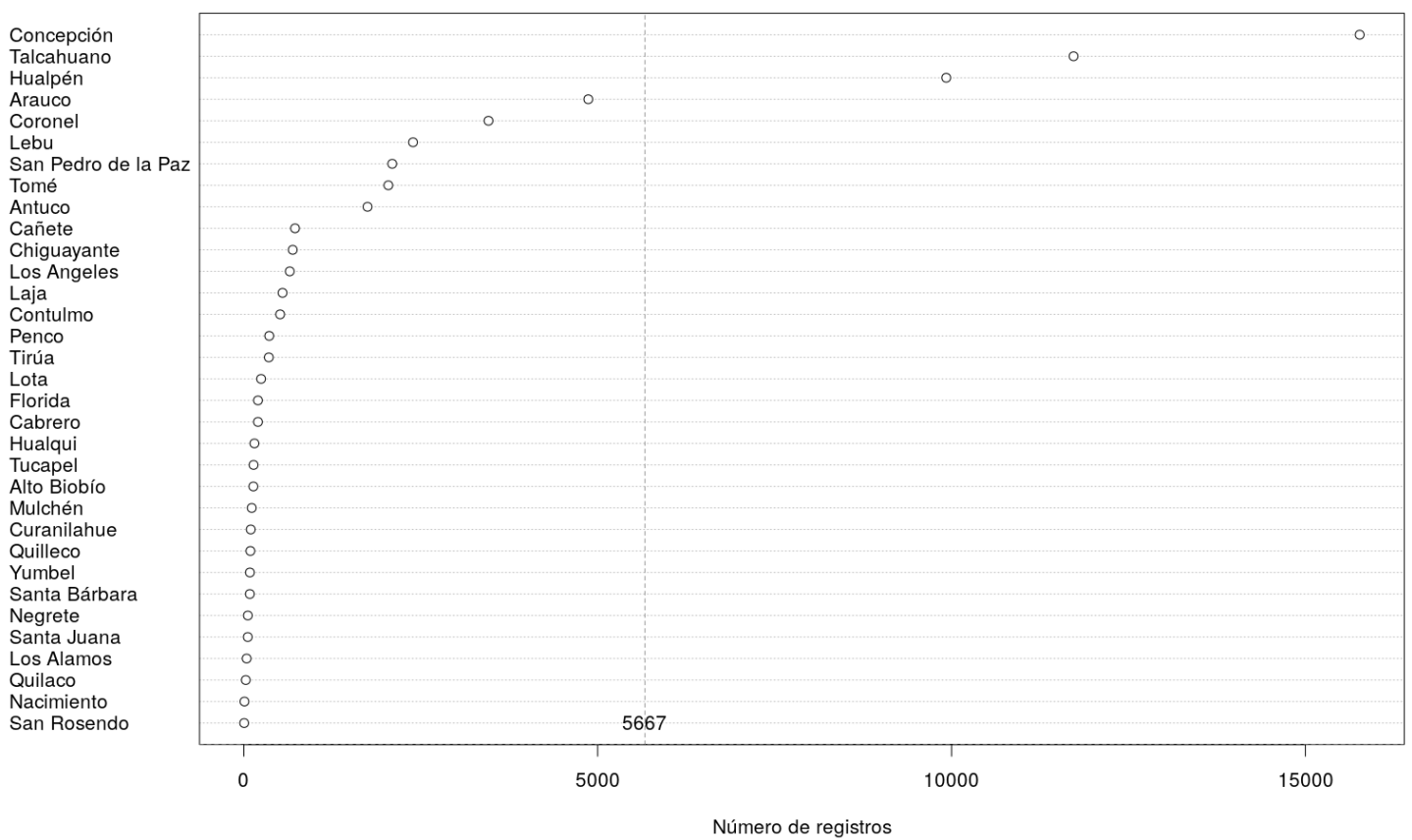

Figura 5. Número de registros de aves por comuna para la Región del Biobío (Chile). La línea en 5667 representa el número de registros necesarios (esfuerzo de muestreo) para obtener un mínimo de $85 \%$ de las aves registradas en toda la región. / Number of bird records per commune for the Biobío Region (Chile). The line at 5,667 represents the number of records needed (sampling effort) to obtain a minimum of $85 \%$ of the birds registered in the entire region.

\section{RIQUEZA ESPECÍFICA (S)}

En Chile, el número de especies de aves varía según la información proporcionada por distintos autores, siendo el rango estimado entre 434 y 498 especies (Araya \& Bernal 1995; Stotz et al. 1996; Araya \& Millie 2000; CONAMA 2008; Stutzin 2009; Sallaberry 2010; Barros et al. 2015). La riqueza específica observada (Sobs) alcanzó un valor de 226 especies, lo que representa entre un 52,1\% y 45,4\% del total de aves registradas en Chile. Mientras que la riqueza específica esperada (Sesp): (a) usando la asíntota (a/b) de la curva de acumulación de especies, fue de 249 especies, lo que representa entre un $57,4 \%$ y $50,0 \%$ del total de especies estimado para Chile; $y$, (b) empleando estimadores no paramétricos varió entre 239 y 249 especies (Tabla 1). La Sobs es menor que el límite superior del rango obtenido por los estimadores no paramétricos en 23,9 especies y, también, menor que el rango inferior en 12,6 especies. La estimación de Sesp, usando la asíntota de la curva de acumulación de especies, se ubica dentro del rango obtenido usando estimadores no paramétricos $\mathrm{y}$, además, concuerda con el valor obtenido por el estimador iChao. Los estimadores no paramétricos, también permitieron evaluar la completitud del inventario, la cual varió entre 90,4 y 94,7, confirmando los resultados obtenidos con la curva de acumulación de especies ajustada.

TABLA 1. Estimadores no paramétricos y su porcentaje de completitud. / Non-parametric estimators and their percentage of completeness.

\begin{tabular}{lcc}
\hline Estimador & Sesp & Completitud (\%) \\
\hline Chao1 & 247,1 & 91,5 \\
iChao1 & 249,9 & 90,4 \\
ACE & 238,6 & 94,7 \\
1st order jackknife & 239,0 & 94,6 \\
2nd order jackknife & 248,0 & 91,1 \\
\hline
\end{tabular}




\section{COMPOSICIÓN DE ESPECIES}

El inventario alcanzó un total de 61.940 registros, que se repartieron en 447 taxa agrupados en 21 Órdenes, 49 Familias, 151 Géneros y 226 Especies de aves (Sobs). El listado con las especies de aves registradas en la Región del Biobío puede ser visto en el Apéndice 1. Se incluye: Orden, Familia, Género, Especie, nombre científico, nombre común, estado de conservación (EC) y endemismo (E). Los taxa más numerosos por el número de especies fueron, entre los Géneros: Calidris con 7 especies, Charadrius con 5 especies y Phalacrocorax con 5 especies; entre las Familias: Anatidae con 19 especies, Furnariidae con 16 especies y Scolopacidae con 16 especies; y, entre los Órdenes: Passeriformes con 69 especies, Charadriiformes con 48 especies, Anseriformes con 19 especies y Procellariiformes con 19 especies. La Fig. 6 muestra una comparación entre Chile y la Región del Biobío, en cuanto al número de categorías taxonómicas. La brecha se acorta a medida que se asciende por los rangos taxonómicos, tanto en número como en proporción.

Distribución del NúMERO de ESPECIES POR COMUNA EN LA REgión DEL Bı́вío

El mapa de la Fig. 7 y el gráfico de la Fig. 8 muestran el número de especies que se han observado en cada una de las comunas. Resulta evidente el amplio rango de variación que va desde 5 especies (comuna de San Rosendo) hasta 173 especies (comuna de Talcahuano). También, resalta la situación de las comunas costeras, ya que poseen los conteos de especies más altos de la región; un caso similar es la comuna de Antuco entre las comunas cordilleranas. Cabe destacar que ninguna de las comunas alcanza 226 especies, que es el número total de especies observado en la región. Talcahuano, la comuna con el mayor número de especies observado, representa una proporción de 76,5\% del total, mientras que San Rosendo, la comuna con menos observaciones, representa sólo un 2,2\% del total.

\section{ESPECIES ENDÉMICAS A NIVEL REGIONAL Y POR COMUNA}

Se observaron seis especies endémicas (ver Apéndice 1), cuya distribución a nivel regional y por comuna se presenta en la Fig. 9. Éstas representan un 2,7\% del total de especies observadas (Sobs $=226$ ) y un $2,4 \%$ del total de especies esperadas (Sesp $=249$, siguiendo el modelo de Clench). En tanto que, representan un $54,6 \%$ del total de especies endémicas observadas tanto en Chile como en el "hotspot" al que pertenece la Región del Biobío ("Chilean Winter RainfallValdivian Forests"). El número de especies endémicas por comuna varía entre 0 y 5 especies (Fig. 10). En general, las comunas costeras concentran un mayor número de especies endémicas por comuna, por ejemplo, Hualpén y Talcahuano poseen 5 especies cada una, Penco y Tomé 4 especies cada una, la excepción es Tirúa que tiene sólo 1 especie endémica, al igual que varias comunas cordilleranas y de interior como: Alto Biobío, Laja, Mulchén, Tucapel y Yumbel, todas con 1 especie endémica. Cabe destacar que las comunas de Los Álamos, Nacimiento, Quillaco, San Rosendo y Santa Juana, carecen de especies endémicas.

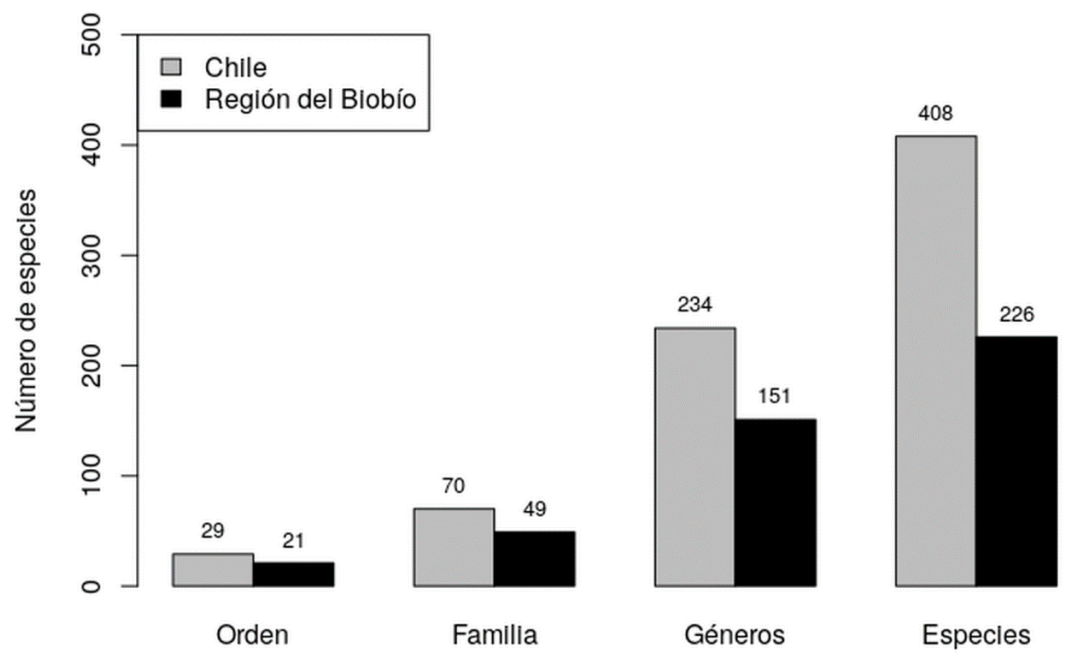

Figura 6. Comparación del número de categorías taxonómicas entre la Región del Biobío y Chile. / Comparison of the number of taxonomic categories between the Biobío Region and Chile. 


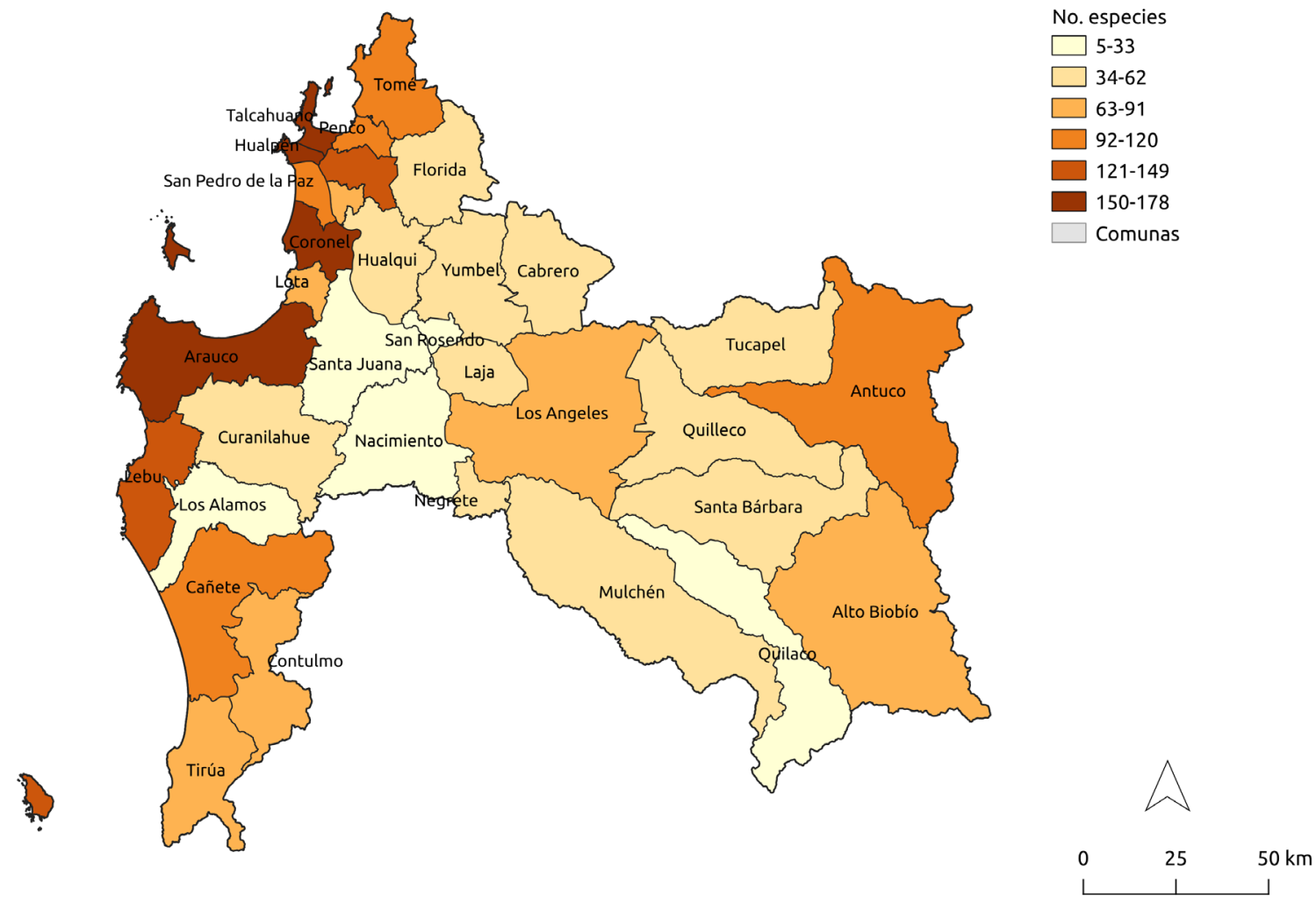

Figura 7. Número de especies por comuna en la Región del Biobío (Chile). / Number of species per commune in the Biobío Region (Chile).

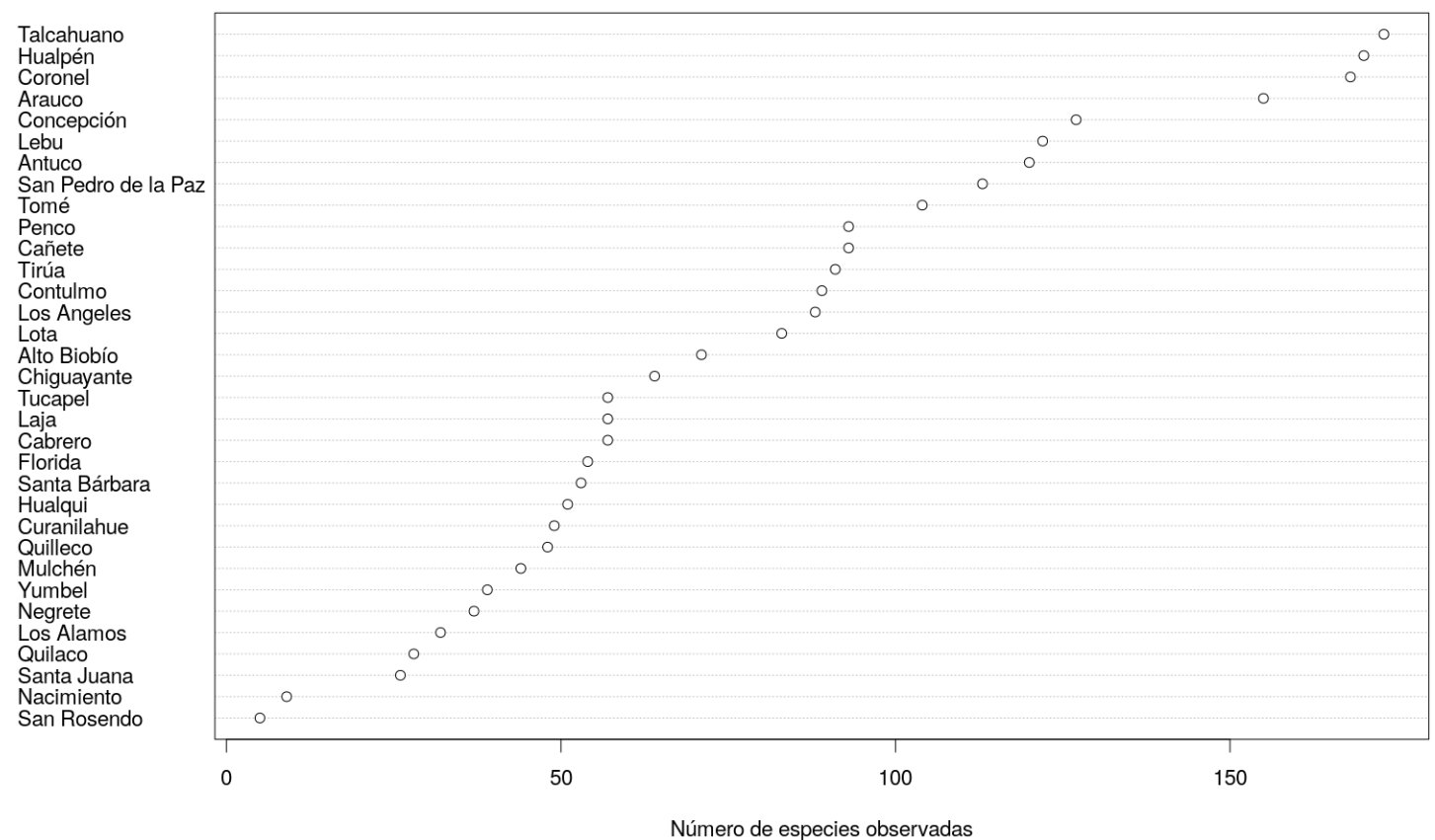

FiguRA 8. Número de especies observadas por comuna (Región del Biobío, Chile). / Number of species observed by commune (Biobío Region, Chile). 


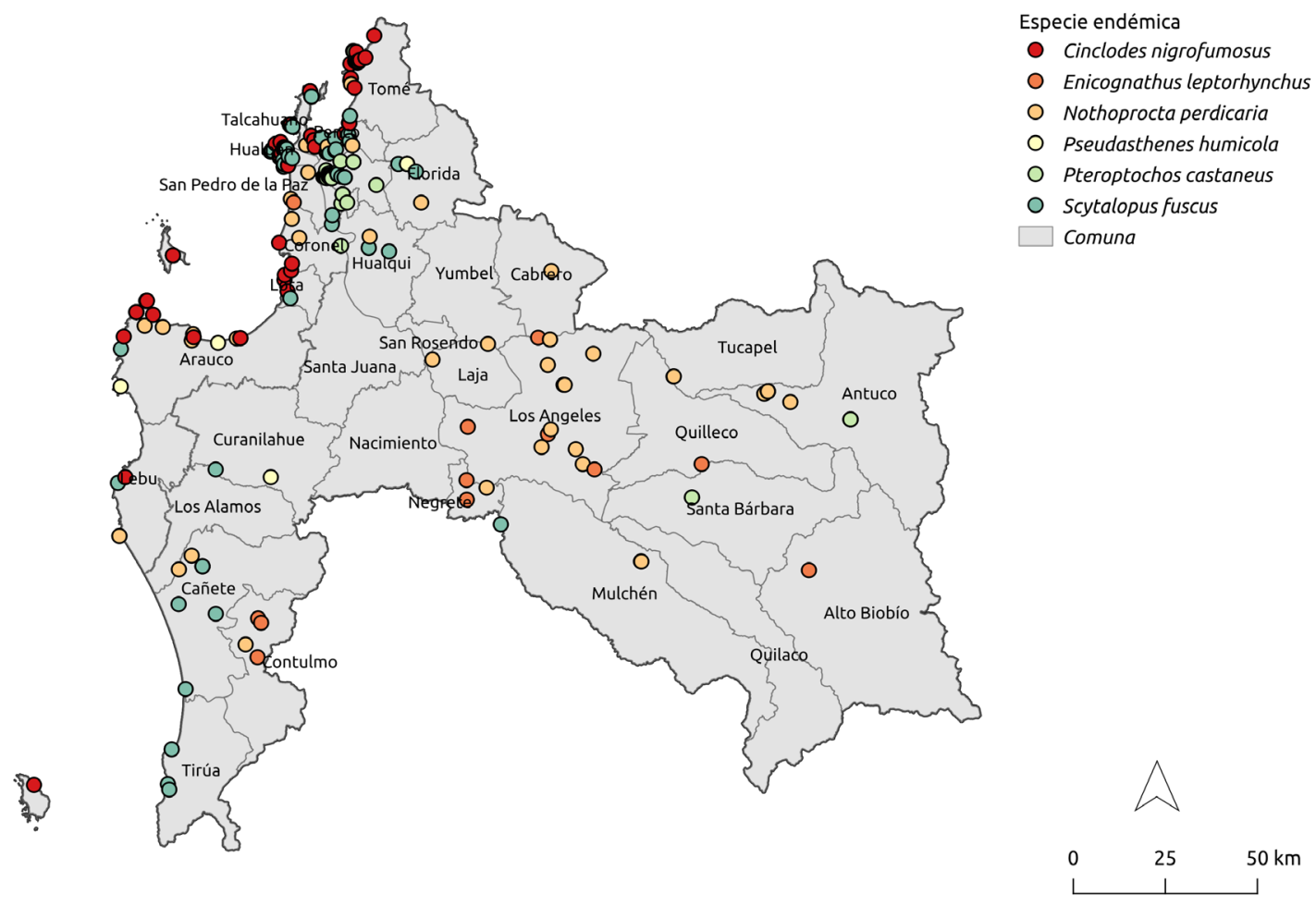

Figura 9. Distribución de las especies endémicas en la Región del Biobío y por comuna. / Distribution of endemic species in the Biobío Region and by commune.

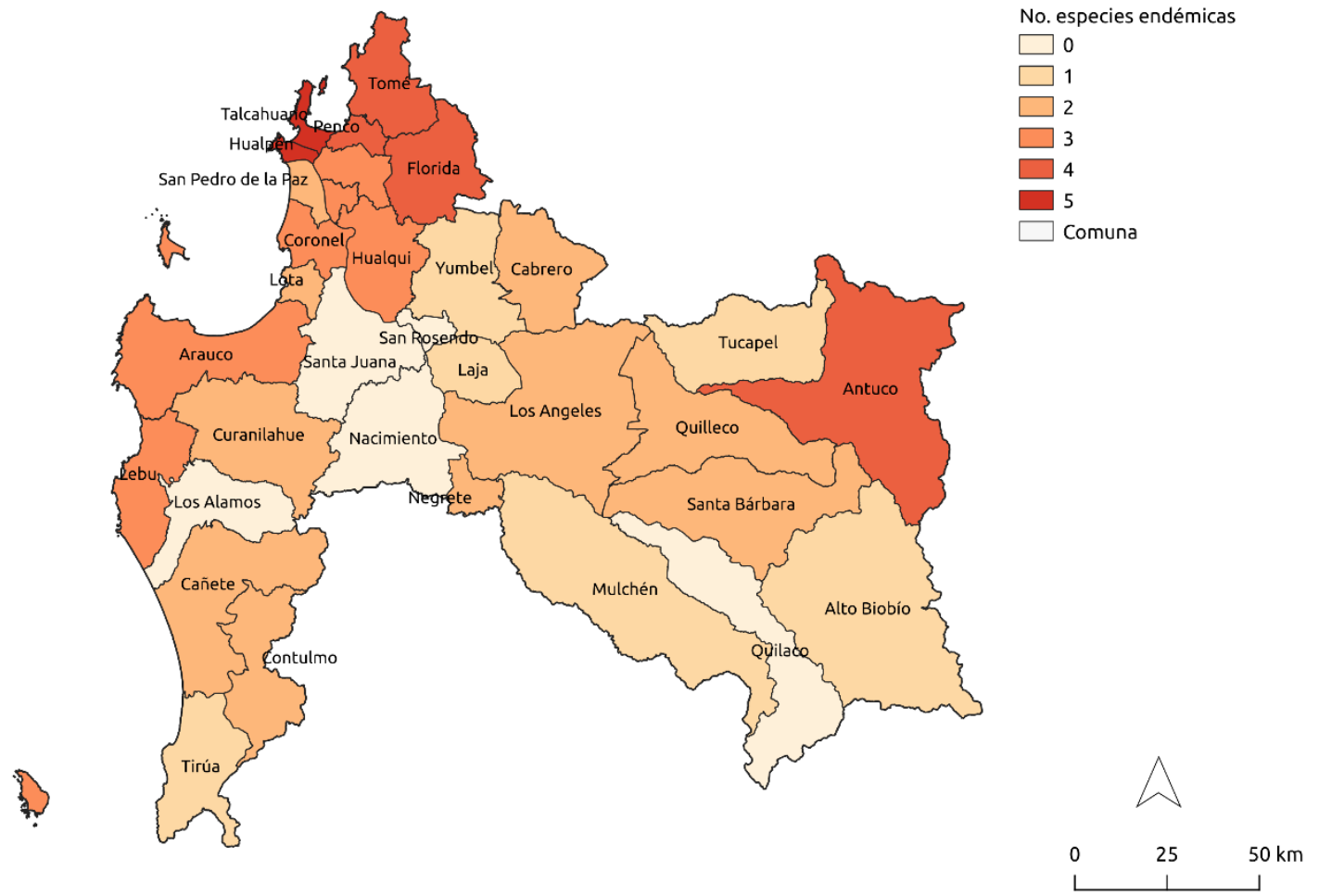

FiguRA 10. Distribución del número de especies endémicas por comuna en la Región del Biobío (Chile). / Distribution of the number of endemic species by commune in the Biobío Region (Chile). 


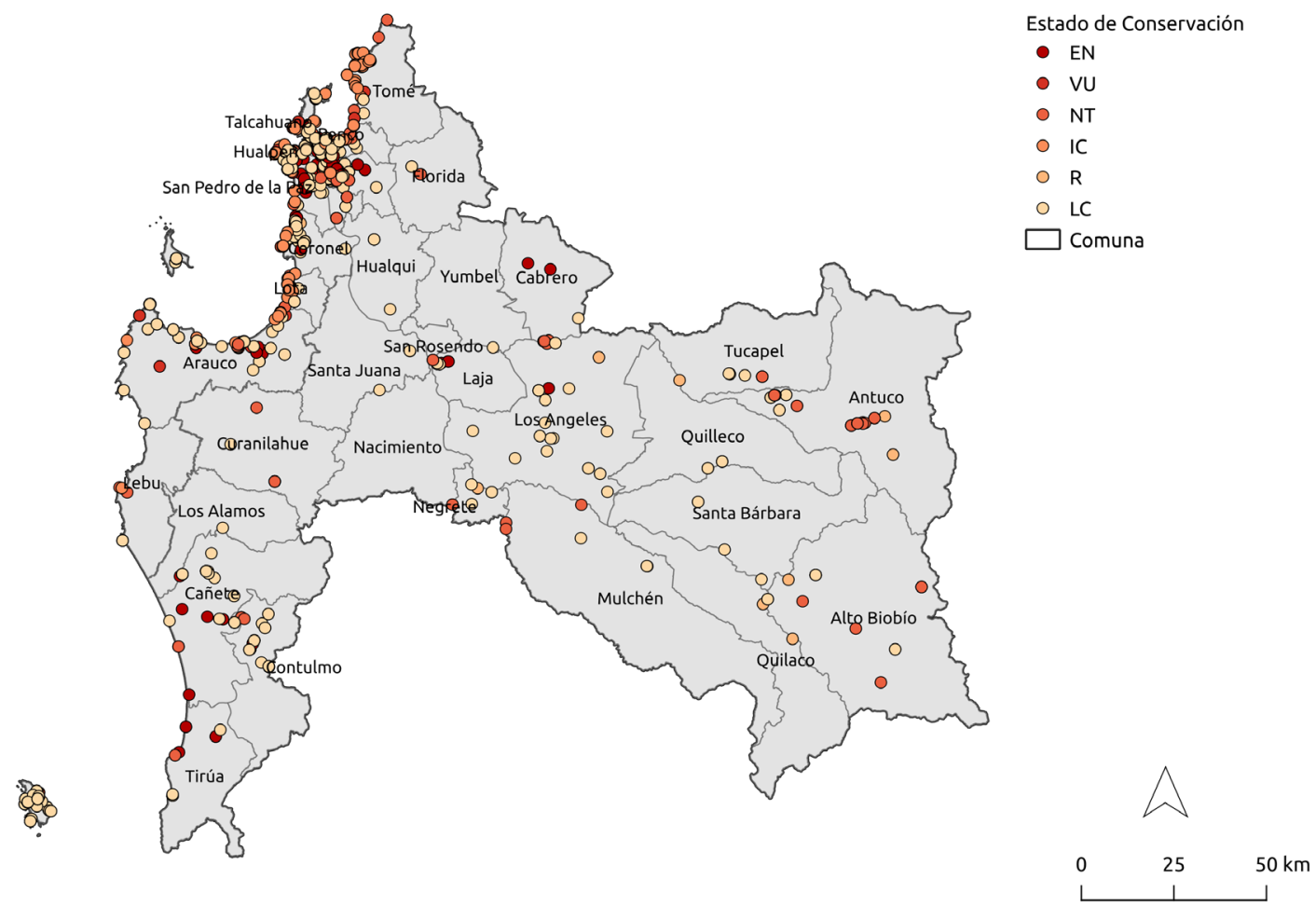

Figura 11. Distribución de las especies amenazadas por estado de conservación en la Región del Biobío y por comuna. EN = En Peligro, VU = Vulnerable, NT = Casi Amenazada, IC = Insuficientemente Conocida, R = Rara, LC = Preocupación Menor. / Distribution of threatened species by conservation status in the Biobío Region and by commune. EN = Endangered, VU = Vulnerable, NT = Near Threatened, IC = Insufficiently Known, R = Rare, LC = Least Concern.

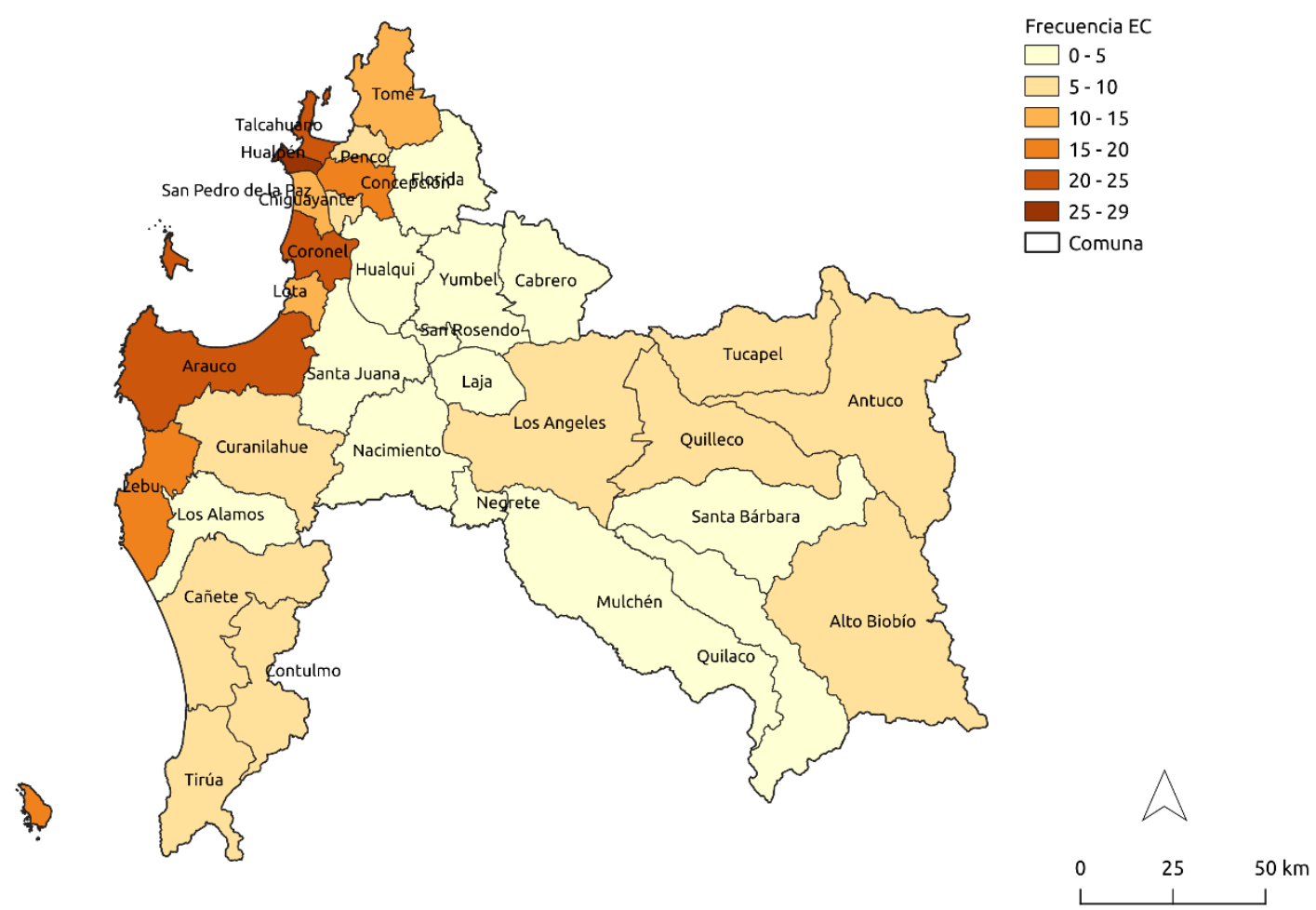

Figura 12. Número de especies amenazadas por comuna en la Región del Biobío. / Number of species threatened by commune in the Biobío Region. 


\section{ESPECIES AMENAZADAS A NIVEL REGIONAL Y POR COMUNA}

En la Región del Biobío se han registrado 37 especies (16,4\% Sobs) con algún grado de amenaza (ver Apéndice 1). El Apéndice 1 muestra el detalle según su estado de conservación (Decreto No29 (D.O. 27/04/2012) del Ministerio del Medio Ambiente), destacando por presentar el mayor número de especies la categoría LC (Preocupación Menor) con 11 especies y por presentar el menor número de especies la categoría IC (Insuficientemente Conocida) con 2 especies. En general, la distribución de las especies amenazadas es irregular en la región (Fig. 11), sin embargo, es posible observar cierta regularidad si consideramos las categorías por separado. Por ejemplo, las especies pertenecientes a la categoría EN (En Peligro), se concentran en comunas principalmente costeras, aun cuando, también es posible observarlas en algunas comunas interiores como Cabrero, Laja y Los Ángeles, además de la cordillerana Tucapel. Otras especies, las pertenecientes a la categoría R (Rara), se agrupan principalmente en las comunas interiores, como Los Ángeles y Negrete, y también en algunas cordilleranas, como Antuco, Alto Biobío y Quilaco. La frecuencia de las especies amenazadas se concentra mayormente en las comunas costeras (Fig. 12). Comunas como Hualpén, Talcahuano, Coronel y Arauco tienen en promedio 25 especies en alguna categoría de conservación; mientras que, comunas más interiores, como Yumbel, Santa Juana, Florida, Hualqui, Laja y Cabrero tienen 3 especies en promedio. Destacan San Rosendo y Nacimiento porque no tienen especies amenazadas.

\section{DISCUSIÓN}

\section{REPRESENTATIVIDAD Y COMPLETITUD DEL INVENTARIO}

El inventario alcanzó un total de 61.940 registros y abarca 47 años de observaciones. El inventario ha resultado ser representativo de la avifauna presente en la Región del Biobío. Usando el cálculo de la pendiente al final de la curva de acumulación ajustada, se obtuvo un valor de 0,004 que es menor a 0,1, lo que indica un inventario completo y fiable, según (Jiménez-Valverde \& Hortal, 2003). Usando tanto el modelo de Clench como estimadores no paramétricos, se superó todos los criterios citados para evaluar la completitud de la curva de acumulación de especies (70\% (JiménezValverde \& Hortal 2003), 85\% (Villarreal et al. 2006) y $90 \%$ (López-Mejía et al. 2017; Moreno \& Halffter 2000). Otra forma de evaluar la utilidad de este inventario es considerando el costo asociado, por ejemplo, aumentar el conocimiento en un $8 \%$, es decir, pasar de la actual completitud de $91 \%$ a una de $99 \%$. Para ello, se requeriría de 37.060 nuevos registros de aves. Esfuerzo de muestreo que, considerando tiempo, presupuesto y personal resulta muy difícil de alcanzar, a través de algún financiamiento formal. Por otro lado, la exploración de nuevas aproximaciones para la estimación del número de especies basadas en taxa superiores, como la propuesta por (Mora et al. 2011), podría contribuir a mejorar los cálculos realizados en este trabajo.

\section{ReGISTROS DE AVES A NIVEL REGIONAL Y POR COMUNA}

A pesar de que, hasta el momento, se ha logrado un inventario representativo de la avifauna regional, con una completitud mayor al 90\%; avanzar hacia otros estudios como la distribución de especies particulares de interés, por ejemplo, aquellas endémicas o amenazadas, debe considerar una evaluación previa de estos datos para integrar este conocimiento en las decisiones de conservación. En efecto, la calidad de los datos no asegura una representación adecuada de los patrones de distribución de las especies dentro de una región (Hortal et al. 2008; Soberón et al. 2000), por lo que es necesario poner atención a las lagunas de información (e.g., sitios con bajo número de especies) y a las variables dependientes de la escala (Soberón et al. 2000), además de, los sesgos espaciales (geográficos), temporales y taxonómicos (Hortal et al. 2008; Soberón et al. 2000). De hecho, a la luz de nuestros resultados, resulta evidente que los registros no se distribuyen aleatoria o regularmente en toda la región y presentarían el patrón de distribución espacial clásico del denominado "síndrome de los recolectores o de las carreteras" ('highway effect'; Hortal et al. 2008; Soberón et al. 2000; Flores-Maldonado et al. 2015;), un sesgo de tipo geográfico descrito como de los más frecuentes en la elaboración de las colecciones biológicas (Flores-Maldonado et al. 2015). Por otro lado, se requiere avanzar hacia la completitud del inventario regional, elaborando inventarios locales centrados en cubrir sitios con lagunas de información como, por ejemplo, las comunas de San Rosendo, Nacimiento y Quilaco (Fig. 5).

\section{RIQUEZA ESPECÍFICA (S)}

La riqueza específica observada (Sobs) alcanzó 226 especies, lo que representa entre un $52,1 \%$ y $45,4 \%$ para el rango de aves totales registradas en Chile (434 y 498 especies, respectivamente). En tanto, la riqueza específica esperada (Sesp), usando el modelo de Clench, alcanzó 249 especies, lo que representa entre un $57,4 \%$ y $50,0 \%$ del rango de especies estimadas para Chile. Y, si consideramos los estimadores no paramétricos, el número de especies esperadas para la región abarca un rango entre 238,6 y 249,9 especies. Cabe destacar que, el valor paramétrico de Sesp (249 especies, ver Fig. 3) coincide con el valor obtenido con el estimador no paramétrico iChao (249,875 especies, ver Tabla 1), posicionando el valor de Sesp más cerca del límite superior del rango obtenido 
entre todos los estimadores. Si consideramos este valor como el esperado para el número de especies de aves en la Región del Biobío, faltarían por descubrirse otras 23 especies.

Otro aspecto de importancia es que la Región del Biobío $\left(36^{\circ} 30^{\prime}-38^{\circ} 30^{\prime} \mathrm{S}\right)$ abarca casi la totalidad del rango donde se observa el mayor número de especies en Chile (Vilina \& Cofré, 2018). La riqueza de especies aumenta hasta alcanzar un máximo en los $36^{\circ}$ de latitud sur y comienza a disminuir a partir de los $39^{\circ}$ de latitud sur, área que corresponde a la transición entre la región Mediterránea y la Templada, esto ocurre entre las Regiones del Maule por el norte y la Araucanía por el sur (Vilina \& Cofré 2018). Por otro lado, esta característica también es compartida por la flora y vegetación de la región ya que aquí se ha descrito un alto nivel de endemismo y una alta riqueza de especies (Fuentes et al. 2017).

\section{COMPOSICIÓN DE ESPECIES}

Si se compara el número de categorías taxonómicas, entre la Región del Biobío y Chile, se observa que la región tiene cada vez mayor participación en el total, en otras palabras, a medida que se asciende por los rangos taxonómicos la proporción se asemeja cada vez más entre ambos territorios (Fig. 6). En efecto, el número de especies observadas en la Región del Biobío representa un 55,4\% del total de especies en Chile, mientras que, el número de Órdenes representa un $72,4 \%$ del total. En general, nuestros resultados son similares a los encontrados por Cursach et al. (2009) para la Región del Maule $\left(34^{\circ}-36^{\circ} \mathrm{S}\right)$. Aunque, en todas las categorías taxonómicas los valores del Maule son levemente menores (Biobío/Maule): 226/213 especies, 151/150 géneros, 21/20 órdenes, excepto para la cantidad de familias, donde ambas regiones presentan el mismo valor (49). A pesar de presentar valores similares, los taxa al interior de cada categoría no son iguales, por ejemplo, dentro de los Órdenes, si bien, la región del Biobío presenta 21 taxa y la del Maule 22, existen 5 órdenes que no son compartidos entre ambas regiones y 18 que sí lo son. En la Región del Biobío se encuentran los Órdenes Accipitriformes, Cathartiformes y Suliformes que no están presentes en la Región del Maule, y en ésta última, se encuentran los Órdenes Apodiformes y Ciconiiformes que no están presentes en la Región del Biobío. Si se observan las Familias ocurre lo mismo. Ambas regiones tienen igual número de Familias (49), pero sólo 44 (89\%) son compartidas (iguales) entre ambas; mientras que, 5 son distintas en cada una de estas regiones siendo, en consecuencia, 10 familias distintas en total. La región del Biobío posee las familias: Oceanitidae, Pandionidae, Passerellidae, Stercorariidae y Thraupidae, que no están presentes en la Región del Maule y esta última posee las familias: Ciconiidae, Emberizidae, Hydrobatidae, Jacanidae y Pelecanoididae, que no están presentes en la Región del Biobío. Esto implica que, en la Región del Biobío, una mayor atención y esfuerzo debe también ponerse en el estudio y conservación de la riqueza de los taxa superiores de aves. Sobre todo, considerando que éstos representan una mayor diversidad filogenética. Como ejemplo, podemos mencionar los géneros Pteroptochos y Scytalopus, indicados por (Vuilleumier, 1985) como los dos únicos casos de especiación actual o potencial detectados en situaciones continentales, ambos representados en la Región del Biobío y centrados en el valle del río Biobío, que puede actuar como barrera para el flujo genético. Para ilustrar mejor aún, pongamos por caso, todos los rinocríptidos que son citados en el trabajo de Willson et al. (1994): Pteroptochos tarnii, Scelorchilus rubecula, Eugralla paradoxa, Scytalopus magellanicus, ya que, según este autor, serían muy sensibles al tamaño de los parches de hábitat, cuya formación a través del proceso de fragmentación es cada vez más preocupante (CONAF 2019; Otavo \& Echeverría 2017). Esta Familia (Rhinocryptidae) está presente en la Región del Biobío (ver Apéndice 1), posee cuatro géneros, pero además de las cuatro especies ya mencionadas, incluye a dos especies más que resultan ser endémicas: Scytalopus fuscus y Pteroptochos castaneus. Es decir, sólo en esta familia se concentran 4 de los 12 géneros existentes a nivel mundial; los 2 géneros indicados como de especiación actual o potencial; junto con 2 especies endémicas que representan el $17 \%$ de las existentes en Chile y el hotspot "Chilean Winter Rainfall - Valdivian Forests".

Esto es importante, porque de acuerdo con (Meffe \& Carroll 1997), los linajes que han evolucionado separadamente por largos períodos de tiempo tienen muchos genes únicos y combinaciones de genes que se pueden perder si estos linajes llegan a extinguirse, además, estos linajes actúan como "almacenes" de información acerca de la historia de la vida que puede ser "leída", usando técnicas moleculares; también, el funcionamiento de los ecosistemas depende, en parte, de la variedad de especies presentes en ellos y, finalmente, los beneficios estéticos que recibimos de la naturaleza están correlacionados con la variedad de organismos vivos con quienes interactuamos, no importa cuántas especies de aves puede haber, ellas no pueden sustituir estéticamente a los mamíferos, peces, reptiles o insectos.

Especies endémicas, raRAs y amenazadas en la Región del Biobío La presencia de 6 especies endémicas en la Región del Biobío se considera alta, dado que alcanza a un $54,6 \%$ tanto del total nacional como del "hotspot" chileno en el que se encuentra inserta la región. Ahora bien, si se considera que el endemismo de aves en Chile es bajo, debido a su alta vagilidad (Victoriano et al. 2006), y a que forman parte de los grupos con más problemas de conservación (Ministerio de Medio 
Ambiente 2020; Spotorno 1995); entonces, las especies de aves endémicas de la Región del Biobío son uno de los grupos prioritarios para su conservación. Además, cabe recordar aquí, lo indicado por (Stotz et al. 1996), sobre que la mayoría de las extinciones en el Neotrópico no ocurren en los centros de alta biodiversidad, sino en centros de endemismo, donde se concentran especies restringidas a hábitats determinados. En este sentido, por ejemplo, los humedales de la Región del Biobío muestran la singularidad y fragilidad de la avifauna acuática, cuando, al mismo tiempo, reúnen en espacios reducidos una alta riqueza de especies y una mayoría de aves especializadas y altamente sensibles a los cambios ambientales (Victoriano et al. 2006). Las especies amenazadas son otro grupo que, por definición, requiere una especial atención, son importantes porque su estado de conservación advierte acerca del riesgo de extinción de sus poblaciones naturales (Decreto N²9 (D.O. 27/04/2012) del Ministerio del Medio Ambiente). En la Región del Biobío se han registrado 37 de ellas (Apéndice 1), de las cuales 6 especies están "En Peligro" (EN), o sea, enfrentan un riesgo muy alto de extinción; 7 especies al ser "Vulnerables" (VU), enfrentan un riesgo alto de extinción; 8 especies pertenecen a la categoría "Casi Amenazada" (NT), es decir, están próximas a incorporarse en las categorías "En Peligro Crítico", "En Peligro" o "Vulnerable"; 11especies pertenecen a la categoría "Preocupación Menor" (LC), es decir, no cumplen con alguno de los criterios que definen las categorías con más riesgo; 2 especies tienen la condición de ser "Insuficientemente Conocidas" (IC); y, 3 son especies "Raras" (R) en la región. Se han incorporado estas últimas, aun cuando no forman parte de alguna categoría de conservación, pero pertenecen a los ensamblajes ecológicos amenazados que deben ser destacados como una prioridad de conservación. Además, no considerar las especies raras significa homogeneizar las diferencias reales entre localidades en cuanto a la diversidad de especies, dado que éstas no se distribuyen aleatoria u homogéneamente en un territorio y, también, porque la riqueza de especies de una localidad suele ser altamente dependiente de la cantidad de especies raras (Hortal \& Lobo 2002).

\section{RELACIÓN CON EL ORDENAMIENTO TERRITORIAL}

La elaboración de estudios científico-biológicos que incluyan objetivos orientados a relacionar sus resultados en un contexto territorial, tal como este, son una forma de expresar las inquietudes científicas y causar un efecto para avanzar en la conservación biológica efectiva y en la integración entre estas disciplinas. Esto se suma a los procesos actuales de participación ciudadana, consulta indígena y Evaluación Ambiental Estratégica correspondientes a los principales mecanismos vigentes para poder opinar e incidir como ciudadanía en el ordenamiento territorial actual de nuestro país (Cross 2016) (Maturana et al. 2017). Se ha sugerido que, para incorporar la biodiversidad a las estrategias territoriales de conservación, la alternativa más racional y rápida sería modelizar la distribución espacial de sus atributos (Hortal \& Lobo 2002), pero ya vimos que antes se requiere poner atención a la calidad de los datos y los sesgos que los afectan (Hortal et al. 2008; Hortal \& Lobo 2002; Soberón et al. 2000).

En la Región del Biobío los criterios prioritarios para la gestión y conservación de las comunidades de aves, que pueden ser incorporados en el Ordenamiento Territorial y derivadas de lo indicado por (Stotz et al. 1996) son: la concentración de especies raras, endémicas, con alguna categoría de riesgo de extinción, restringidas a uno o pocos hábitats y sensibles a las perturbaciones antrópicas. Sin embargo, este conocimiento por sí mismo no asegura la conservación de las aves, también es necesaria (y urgente) la divulgación de su conocimiento entre quienes realizan la planificación y gestión del territorio regional, puesto que este conjunto de acciones (y decisiones) afecta y regula no sólo el espacio físico, sino también, los procesos y la dinámica del territorio ocupado por la avifauna.

\section{AGRADECIMIENTOS}

Estudio auto-financiado por el autor principal y coautor Ad Honorem. Se agradece la participación a lo largo de todo este trabajo del Dr. Heraldo Norambuena, Universidad de Concepción, quién a través de sus consejos, contribuciones, bibliografía e información ayudó a elaborar este artículo. También, a la profesora Patricia Virano Reyes, Universidad de Concepción, por su ayuda y consejos en el manejo de QGis. Y, por la provisión de los datos que sustentan este trabajo, a eBird-Chile y la ROC.

\section{REFERENCIAS}

Araya, B., Bernal, M. 1995. Aves. En: Simonetti, J.A., Arroyo, M.T., Sportono, A.E., Lozada, E. (Eds.) Diversidad Biológica de Chile: 350-360. Comité Nacional de Investigación Científica y Tecnológica, Chile.

Araya, B., Millie, G. 2000. Guía de campo de las aves de Chile. Editorial Universitaria, Chile.

Arroyo, M.T., Marquet, P.A., Marticorena, C., Cavieres, L.A., Squeo, F.A., Simonetti Zambelli, J.A., Rozzi, R., Massardo, F. 2006. El hotspot chileno, prioridad mundial para la conservación. Diversidad de ecosistemas, ecosistemas terrestres. En: 
Biodiversidad de Chile: patrimonio y desafío: 94-99. Comisión Nacional del Medio Ambiente, Chile. URL: http://repositorio.uchile.cl/handle/2250/120068

Barros, R., Jaramillo, Á., Schmitt, F. 2015. Lista de las Aves de Chile 2014. La Chiricoca 20: 79-100.

Berdegué, J., Jara, E., Modrego, F., Sanclemente, X., Schejtman, A. 2010. Comunas rurales de Chile. Documento de Trabajo N 60; Programa Dinámicas Territoriales Rurales. Rimisp.

Biblioteca del Congreso Nacional. s.f. Biblioteca del Congreso Nacional, SIIT, Chile Nuestro País. bcn.cl. Accedido: Diciembre 17, 2019. URL: https://www.bcn.cl/siit/ nuestropais/index_html

Carrasco Lagos, P. 2004. Variaciones de la estructura del ensamble de aves a lo largo de un gradiente ambiental en el humedal Tubul-raqui, VIII Región, Chile. PhD Thesis. Universidad de Concepción, Chile.

Chao, A., Ma, K.H., Hsieh, T.C., Chiu, C.-H. 2016. SpadeR: Species-Richness Prediction and Diversity Estimation with R. https://CRAN.R-project.org/package=SpadeR

Clements. 2019. The Clements Checklist of Birds of the World. Accedido: Diciembre 19, 2019. URL: https://www.birds. cornell.edu/clementschecklist/?__hstc $=43953530.630 \mathrm{a}$ 94281b874b0eb1d04c9ce5d567a9.1576776227404. $1576776227404.1576776227404 .1 \&_{-}$hssc $=43953$ 530.1.1576776227406\&__hsfp=98972621

CONAF. 2019. Catastro Vegetacional. URL: http://www.conaf.cl/ nuestros-bosques/bosques-en-chile/catastrovegetacional/

CONAMA. 2008. Biodiversidad de Chile, Patrimonio y Desafíos. Segunda Edición. Ocho Libros Editores, Chile.

Cross, V. 2016. Ordenamiento territorial en Chile: ¿cómo se está planificando nuestro territorio? Ladera Sur. URL: https:// laderasur.com/mas/ordenamiento-territorial-en-chilecomo-se-esta-planificando-nuestro-territorio

Cursach, J., Rau, J., Suazo, C. 2009. Sinopsis sobre el conocimiento de las aves en la región del Maule, Chile central. Boletín Chileno de Ornitología 15(2): 57-72.

De Mas, E. 2007. Evaluación y predicción de la biodiversidad. Un modelo con Araneidos en el Parque Natural del CadíMoixeró. Tesis Doctoral. Universitat de Barcelona, España. eBird. 2019. eBird Basic Dataset. Cornell Lab of Ornithology.

Feria-Arroyo, T.P., Sánchez-Rojas, G., Ortiz-Pulido, R., BravoCadena, J., Calixto Pérez, E., Dale, J.M., Duberstein, J.N., Lara, C., Valencia-Herverth, J. 2013. Estudio del cambio climático y su efecto en las aves en México: enfoques actuales y perspectivas futuras. Huitzil 14(1): 47-55

Figueroa, R.A., Bravo, C., Corales, E.S., López, R., Alvarado, S. 2000. Avifauna del Santuario de la Naturaleza Los Huemules del Niblinto, Región del Biobío, Chile. Boletín Chileno de Ornitología 7: 2-12.
Flores-Maldonado, J.J., Ruvalcaba-Ortega, I., Moreno-Talamantes, A., García-Aranda, M. A., Favela-Lara, S., González-Rojas, J.I. 2015. Representatividad geográfica y ambiental del inventario de especies arbustivas en el Área de Protección de Recursos Naturales" Cuenca Alimentadora del Distrito Nacional de Riego 004 Don Martín", Coahuila, México. Revista Mexicana de Biodiversidad 86(3): 809-822.

Fuentes, G., Cisternas, A., Valencia, G., Mihoc, M., Ruiz, E., Hasbún, R., Valenzuela, S., Baeza, C.M. 2017. Diversidad taxonómica y genética del sitio prioritario Península de Hualpén, Región del Bío-Bío, Chile. Implicancias para la conservación. Gayana Botánica 74(1): 94-110. https://doi. org/10.4067/S0717-66432017005000112

Fuenzalida, H., Pisano, E. 1965. Biogeografía. Geografía Económica de Chile. Texto refundido. Ed. de la Corporación de Fomento.

González, A.L., Vukasovic, M.A., Estades, C.F. 2011. Variación temporal en la abundancia y diversidad de aves en el humedal del Río Itata, región del Bío-Bío, Chile. Gayana 75(2): 170-181. https://doi.org/10.4067/S0717-653820 11000200006

Halffter, G., Moreno, C.E., Pineda, E.O. 2001. Manual para evaluación de la biodiversidad en Reservas de la Biosfera. CYTED, ORCYT/UNESCO \& SEA.

Hortal, J., Jiménez-Valverde, A., Gómez, J.F., Lobo, J.M., Baselga, A. 2008. Historical bias in biodiversity inventories affects the observed environmental niche of the species. Oikos 117(6): 847-858.

Hortal, J., Lobo, J.M. 2002. Una metodología para predecir la distribución espacial de la diversidad biológica. Ecología 16: 405-432.

Informe del estado del medio ambiente 2016 (p. 553). 2016. Ministerio del Medio Ambiente. https://sinia.mma.gob.cl/ wp-content/uploads/2017/08/IEMA2016.pdf

Jiménez, M. 1999. Evaluación del estado de conservación de las aves de humedal de la Región del Biobío. Comisión Nacional del Medio Ambiente, Región del Biobío, Chile.

Jiménez-Valverde, A., Hortal, J. 2003. Las curvas de acumulación de especies y la necesidad de evaluar la calidad de los inventarios biológicos. Revista ibérica de aracnología 8: 151-161.

Lobo, J.M., Martin-Piera, F. 2002. Searching for a predictive model for species richness of Iberian dung beetle based on spatial and environmental variables. Conservation Biology 16(1): 158-173. https://doi.org/10.1046/j.15231739.2002.00211.x

López-Mejía, M., Moreno, C.E., Zuria, I., Sánchez-Rojas, G., Rojas-Martínez, A. 2017. Comparación de dos métodos para analizar la proporción de riqueza de especies entre comunidades: un ejemplo con murciélagos de 
selvas y hábitats modificados. Revista Mexicana de Biodiversidad 88(1): 183-191. https://doi.org/10.1016/j. rmb.2017.01.008

Maturana, F., Fuenzalida, M., Arenas, F., Henríquez, C. 2017. La planificación territorial en Chile y el proceso de descentralización. En: Vial, C., Hernández, J. (Eds.) ¿Para qué descentralizar? Centralismo y políticas públicas en Chile: Análisis y evaluación por sectores: 181-208. ICHEM. URL: https://www.researchgate.net/ publication/328662800_La_planificacion_territorial_en_ Chile_y_el_proceso_de_descentralizacion

Medrano, F., Barros, R., Norambuena, H., Matus, R., Schmitt, F. 2018. Atlas de las aves nidificantes de Chile. Red de Observadores de Aves y Vida Silvestre de Chile. Santiago.

Meffe, G.K., Carroll, C.R. 1997. Principles of conservation biology. 2nd edition. Sinauer Associates, Inc.

Mella, J.E., Simonetti, J.A., Sportono, A.E., Contreras, L.C. 2002. Mamíferos de Chile. En: Ceballos, G., Simonetti, J.A. (Eds.) Diversidad y conservación de los mamíferos neotropicales. CONABIO-UNAM. URL: https://www.researchgate.net/ publication/329481178_MAMIFEROS_DE_CHILE

Ministerio de Medio Ambiente. 2020. Clasificación de Especies Clasificación según estado de conservación. URL: https:// clasificacionespecies.mma.gob.cl/

Mora, C., Tittensor, D.P., Adl, S., Simpson, A.G.B., Worm, B. 2011. How many species are there on earth and in the ocean? PLOS Biology 9(8): e1001127. https://doi.org/10.1371/ journal.pbio.1001127

Moreno, C.E. 2001. Métodos para medir la biodiversidad. Vol. 1. M\&T - Manuales y Tesis SEA.

Moreno, C.E., Halffter, G. 2000. Assessing the completeness of bat biodiversity inventories using species accumulation curves. Journal of Applied Ecology 37(1): 149-158. https://doi.org/10.1046/j.1365-2664.2000.00483.x

Myers, N., Mittermeier, R.A., Mittermeier, C.G., da Fonseca, G.A.B., Kent, J. 2000. Biodiversity hotspots for conservation priorities. Nature 403(6772): 853-858. https://doi.org/10.1038/35002501

Oksanen, J., Blanchet, F.G., Friendly, M., Kindt, R., Legendre, P., McGlinn, D., Minchin, P.R., O'Hara, R.B., Simpson, G.L., Solymos, P., Stevens, M.H.H., Szoecs, E., Wagner, H. 2019. vegan: Community Ecology Package. https:// CRAN.R-project.org/package=vegan

Otavo, S., Echeverría, C. 2017. Fragmentación progresiva y pérdida de hábitat de bosques naturales en uno de los hotspot mundiales de biodiversidad. Revista Mexicana de Biodiversidad 88(4): 924-935. https://doi.org/10.1016/j. rmb.2017.10.041

QGIS Development Team. 2015. QGIS Geographic Information System. Open Source Geospatial Foundation Project. http://qgis.osgeo.org

R Core Team. 2019. R: A Language and Environment for Statistical Computing. R Foundation for Statistical Computing. https://www.R-project.org/

Riffo, R., Villarroel, C. 2000. Caracterización de la flora y fauna del humedal Los Batros, comuna de San Pedro de la Paz. Gayana 64: 23-37.

Riquelme, C., Hinojosa, A., Figueroa, R.A., González-Acuña, D. 2018. Aves de la Reserva Nacional Isla Mocha, sur de Chile: Riqueza, abundancia y diversidad de especies. Gayana 82(1): 40-64. https://doi.org/10.4067/S071765382018000100040

RStudio Team. 2018. RStudio: Integrated Development Environment for R. RStudio, Inc. https://www.rstudio.com/

Sallaberry, M. 2010. Aves marinas. Bibliografía sobre biodiversidad acuática de Chile: 435-448. Comité Oceanográfico Nacional. URL: http://www.cona.cl/pub/ libro_bibliografia/35.PDF

Soberón, J., Llorente, J. 1993. The use of species accumulation functions for the prediction of species richness. Conservation Biology 7(3): 480-488. https://doi. org/10.1046/j.1523-1739.1993.07030480.x

Soberón, J.M., Llorente, J.B., Oñate, L. 2000. The use of specimen-label databases for conservation purposes: an example using Mexican Papilionid and Pierid butterflies. Biodiversity \& Conservation 9(10): 1441-1466. https:// doi.org/10.1023/A:1008987010383

Spotorno, A.E. 1995. Vertebrados. En: Simonetti, J.A., Sportono, A.E., Lozada, E. (Eds.) Diversidad Biológica de Chile: 299-301. Comité Nacional de Investigación Científica y Tecnológica, Chile.

StatSoft Inc. 2007. STATISTICA (data analysis software system), version 8.0. http://www.statsoft.com

Stotz, D.F., Fitzpatrick, J.W., III, T.A.P., Moskovits, D.K. 1996. Neotropical Birds: Ecology and Conservation. University of Chicago Press.

Stutzin, M. 2009. Cuarto informe nacional de biodiversidad. Convenio sobre Diversidad Biológica: 140. Comisión Nacional del Medio Ambiente, Chile.

Subdere. s.f. BIOBIO - Estrategia Regional de Desarrollo Periodo 2015-2030. Accedido: Junio 16, 2020. URL: http:// www.subdere.gov.cl/documentacion/biobio-estrategiaregional-de-desarrollo-periodo-2015-2030

Tejeda, I., Medrano, F. 2018. eBird como una herramienta para mejorar el conocimiento de las aves de Chile. Revista Chilena de Ornitología 24(2): 85-94.

Victoriano, P.F., González, A.L., Schlatter, R. 2006. Estado de conocimiento de las aves de aguas continentales de Chile. Gayana 70(1): 140-162. https://doi.org/10.4067/S071765382006000100019 
Vilina, Y., Cofré, H. 2018. Aves Terrestres. En: Biodiversidad de Chile. Patrimonio y Desafíos: 246-251. Tercera Edición. Vol. 1. Ministerio del Medio Ambiente, Chile.

Villarreal, H., Álvarez, M., Córdoba, S., Escobar, F., Fagua, G., Gast, F., Mendoza, H., Ospina, M., Umaña, A. 2006. Manual de métodos para el desarrollo de inventarios de biodiversidad. Programa de Inventarios de Biodiversidad. Segunda edición. Instituto de Investigación de Recursos
Biológicos Alexander von Humboldt.

Vuilleumier, F. 1985. Forest birds of Patagonia: ecological geography, speciation, endemism, and faunal history: 255-304. Ornithological Monographs.

Willson, M.F., De Santo, T.L., Sabag, C., Armesto, J.J. 1994. Avian Communities of Fragmented South-Temperate Rainforests in Chile. Conservation Biology 8(2): 508-520. https://doi.org/10.1046/j.1523-1739.1994.08020508

Received: 16.07 .2020

Accepted: 03.05.2021

ApÉndiCe 1. Listado de especies de aves de la Región del Biobío. Se incluye: Orden, Familia, Género, Nombre científico, Nombre común, Estado de Conservación (EC) y Endemismo (E). Estado de Conservación: EN = En Peligro, VU = Vulnerable, NT = Casi amenazada, LC =Preocupación Menor, IC = Insuficientemente Conocida, R = Rara. Endémica: * . / List of bird species in the Biobío Region. Includes: Order, Family, Gender, Scientific name, Common name, Conservation Status (EC) and Endemism (E). Conservation Status: EN = Endangered, VU = Vulnerable, NT = Near Threatened, LC = Least Concern, IC = Insufficiently Known, R = Rare. Endemic: *.

\begin{tabular}{|c|c|c|c|c|c|c|}
\hline Orden & Familia & Género & Nombre científico & Nombre común & EC & $\mathbf{E}$ \\
\hline Accipitriformes & Accipitridae & Accipiter & Accipiter bicolor & Peuquito & $\mathrm{R}$ & \\
\hline Passeriformes & Icteridae & Agelasticus & Agelasticus thilius & Trile & & \\
\hline Passeriformes & Tyrannidae & Agriornis & Agriornis lividus & Mero común & & \\
\hline Passeriformes & Tyrannidae & Agriornis & Agriornis montanus & Mero gaucho & & \\
\hline Passeriformes & Tyrannidae & Agriornis & Agriornis murinus & Mero chico & & \\
\hline Passeriformes & Tyrannidae & Anairetes & Anairetes parulus & Cachudito & & \\
\hline Anseriformes & Anatidae & Anas & Anas bahamensis & Pato gargantillo & LC & \\
\hline Anseriformes & Anatidae & Anas & Anas flavirostris & Pato jergón chico & & \\
\hline Anseriformes & Anatidae & Anas & Anas georgica & Pato jergón grande & & \\
\hline Passeriformes & Motacillidae & Anthus & Anthus correndera & Bailarín chico & & \\
\hline Passeriformes & Motacillidae & Anthus & Anthus hellmayri & Bailarín chico argentino & & \\
\hline Passeriformes & Furnariidae & Aphrastura & Aphrastura spinicauda & Rayadito & & \\
\hline Pelecaniformes & Ardeidae & Ardea & Ardea alba & Garza grande & & \\
\hline Pelecaniformes & Ardeidae & Ardea & Ardea cocoi & Garza cuca & LC & \\
\hline Procellariiformes & Procellariidae & Ardenna & Ardenna bulleri & Fardela de dorso gris & & \\
\hline Procellariiformes & Procellariidae & Ardenna & Ardenna creatopus & Fardela blanca & EN & \\
\hline Procellariiformes & Procellariidae & Ardenna & Ardenna grisea & Fardela negra & NT & \\
\hline Charadriiformes & Scolopacidae & Arenaria & Arenaria interpres & Playero vuelvepiedras & & \\
\hline Strigiformes & Strigidae & Asio & Asio flammeus & Nuco & LC & \\
\hline Passeriformes & Furnariidae & Asthenes & Asthenes anthoides & Canastero del Sur & & \\
\hline Passeriformes & Furnariidae & Asthenes & Asthenes pyrrholeuca & Canastero de cola larga & & \\
\hline Strigiformes & Strigidae & Athene & Athene cunicularia & Pequén & & \\
\hline Strigiformes & Strigidae & Bubo & Bubo virginianus & Tucúquere & & \\
\hline Pelecaniformes & Ardeidae & Bubulcus & Bubulcus ibis & Garza boyera & & \\
\hline Accipitriformes & Accipitridae & Buteo & Buteo albigula & Aguilucho chico & $\mathrm{R}$ & \\
\hline
\end{tabular}




\begin{tabular}{|c|c|c|c|c|c|c|}
\hline Orden & Familia & Género & Nombre científico & Nombre común & EC & E \\
\hline Accipitriformes & Accipitridae & Buteo & Buteo ventralis & Aguilucho de cola rojiza & VU & \\
\hline Charadriiformes & Scolopacidae & Calidris & Calidris alba & Playero blanco & & \\
\hline Charadriiformes & Scolopacidae & Calidris & Calidris bairdii & Playero de Baird & & \\
\hline Charadriiformes & Scolopacidae & Calidris & Calidris canutus & Playero ártico & & \\
\hline Charadriiformes & Scolopacidae & Calidris & Calidris melanotos & Playero pectoral & & \\
\hline Charadriiformes & Scolopacidae & Calidris & Calidris minutilla & Playero enano & & \\
\hline Charadriiformes & Scolopacidae & Calidris & Calidris pusilla & Playero semipalmado & & \\
\hline Charadriiformes & Scolopacidae & Calidris & Calidris virgata & Playero de las rompientes & & \\
\hline Galliformes & Odontophoridae & Callipepla & Callipepla californica & Codorniz & & \\
\hline Piciformes & Picidae & Campephilus & $\begin{array}{l}\text { Campephilus } \\
\text { magellanicus }\end{array}$ & Carpintero negro & VU & \\
\hline Falconiformes & Falconidae & Caracara & Caracara plancus & Traro & & \\
\hline Cathartiformes & Cathartidae & Cathartes & Cathartes aura & Jote de cabeza colorada & & \\
\hline Charadriiformes & Charadriidae & Charadrius & Charadrius collaris & Chorlo de collar & & \\
\hline Charadriiformes & Charadriidae & Charadrius & Charadrius falklandicus & Chorlo de doble collar & & \\
\hline Charadriiformes & Charadriidae & Charadrius & Charadrius modestus & Chorlo chileno & & \\
\hline Charadriiformes & Charadriidae & Charadrius & Charadrius nivosus & Chorlo nevado & & \\
\hline Charadriiformes & Charadriidae & Charadrius & $\begin{array}{l}\text { Charadrius } \\
\text { semipalmatus }\end{array}$ & Chorlo semipalmado & & \\
\hline Anseriformes & Anatidae & Chloephaga & Chloephaga hybrida & Caranca & & \\
\hline Anseriformes & Anatidae & Chloephaga & Chloephaga picta & Caiquén & & \\
\hline Anseriformes & Anatidae & Chloephaga & $\begin{array}{l}\text { Chloephaga } \\
\text { poliocephala }\end{array}$ & Canquén & & \\
\hline Charadriiformes & Laridae & Chroicocephalus & $\begin{array}{l}\text { Chroicocephalus } \\
\text { maculipennis }\end{array}$ & Gaviota cáhuil & & \\
\hline Charadriiformes & Laridae & Chroicocephalus & $\begin{array}{l}\text { Chroicocephalus } \\
\text { serranus }\end{array}$ & Gaviota andina & $\mathrm{R}$ & \\
\hline Passeriformes & Furnariidae & Cinclodes & Cinclodes fuscus & Churrete acanelado & & \\
\hline Passeriformes & Furnariidae & Cinclodes & Cinclodes nigrofumosus & Churrete costero & & * \\
\hline Passeriformes & Furnariidae & Cinclodes & Cinclodes oustaleti & Churrete chico & & \\
\hline Passeriformes & Furnariidae & Cinclodes & Cinclodes patagonicus & Churrete & & \\
\hline Accipitriformes & Accipitridae & Circus & Circus cinereus & Vari & & \\
\hline Passeriformes & Troglodytidae & Cistothorus & Cistothorus platensis & Chercán de las vegas & & \\
\hline Piciformes & Picidae & Colaptes & Colaptes pitius & Pitío & & \\
\hline Passeriformes & Tyrannidae & Colorhamphus & $\begin{array}{l}\text { Colorhamphus } \\
\text { parvirostris }\end{array}$ & Viudita & & \\
\hline Columbiformes & Columbidae & Columba & Columba livia & Paloma doméstica & & \\
\hline Columbiformes & Columbidae & Columbina & Columbina picui & Tortolita cuyana & & \\
\hline Cathartiformes & Cathartidae & Coragyps & Coragyps atratus & Jote de cabeza negra & & \\
\hline Anseriformes & Anatidae & Coscoroba & Coscoroba coscoroba & Cisne coscoroba & EN & \\
\hline Passeriformes & Icteridae & Curaeus & Curaeus curaeus & Tordo & & \\
\hline Anseriformes & Anatidae & Cygnus & Cygnus melancoryphus & Cisne de cuello negro & EN & \\
\hline
\end{tabular}




\begin{tabular}{|c|c|c|c|c|c|c|}
\hline Orden & Familia & Género & Nombre científico & Nombre común & EC & $\mathrm{E}$ \\
\hline Procellariiformes & Procellariidae & Daption & Daption capense & Petrel damero & & \\
\hline Procellariiformes & Diomedeidae & Diomedea & Diomedea epomophora & Albatros real del sur & & \\
\hline Passeriformes & Thraupidae & Diuca & Diuca diuca & Diuca & & \\
\hline Passeriformes & Icteridae & Dolichonyx & Dolichonyx oryzivorus & Charlatán & & \\
\hline Piciformes & Picidae & Dryobates & Dryobates lignarius & Carpinterito & & \\
\hline Pelecaniformes & Ardeidae & Egretta & Egretta caerulea & Garza azul & & \\
\hline Pelecaniformes & Ardeidae & Egretta & Egretta thula & Garza chica & & \\
\hline Passeriformes & Tyrannidae & Elaenia & Elaenia albiceps & Fio-fio & & \\
\hline Accipitriformes & Accipitridae & Elanus & Elanus leucurus & Bailarín & & \\
\hline Psittaciformes & Psittacidae & Enicognathus & $\begin{array}{l}\text { Enicognathus } \\
\text { ferrugineus }\end{array}$ & Cachaña & & \\
\hline Psittaciformes & Psittacidae & Enicognathus & $\begin{array}{l}\text { Enicognathus } \\
\text { leptorhynchus }\end{array}$ & Choroy & $\mathrm{LC}$ & * \\
\hline Passeriformes & Rhinocryptidae & Eugralla & Eugralla paradoxa & Churrín de la Mocha & & \\
\hline Falconiformes & Falconidae & Falco & Falco femoralis & Halcón perdiguero & & \\
\hline Falconiformes & Falconidae & Falco & Falco peregrinus & Halcón peregrino & & \\
\hline Falconiformes & Falconidae & Falco & Falco sparverius & Cernícalo & & \\
\hline Gruiformes & Rallidae & Fulica & Fulica armillata & Tagua & & \\
\hline Gruiformes & Rallidae & Fulica & Fulica leucoptera & Tagua chica & & \\
\hline Gruiformes & Rallidae & Fulica & Fulica rufifrons & Tagua de frente roja & & \\
\hline Procellariiformes & Procellariidae & Fulmarus & Fulmarus glacialoides & Petrel plateado & & \\
\hline Charadriiformes & Scolopacidae & Gallinago & Gallinago paraguaiae & Becacina & & \\
\hline Passeriformes & Furnariidae & Geositta & Geositta cunicularia & Minero & & \\
\hline Passeriformes & Furnariidae & Geositta & Geositta rufipennis & Minero cordillerano & & \\
\hline Passeriformes & Thraupidae & Geospizopsis & Geospizopsis unicolor & Pájaro plomo & & \\
\hline Accipitriformes & Accipitridae & Geranoaetus & $\begin{array}{l}\text { Geranoaetus } \\
\text { melanoleucus }\end{array}$ & Águila & & \\
\hline Accipitriformes & Accipitridae & Geranoaetus & Geranoaetus polyosoma & Aguilucho & & \\
\hline Strigiformes & Strigidae & Glaucidium & Glaucidium nana & Chuncho austral & & \\
\hline Charadriiformes & Haematopodidae & Haematopus & Haematopus ater & Pilpilén negro & & \\
\hline Charadriiformes & Haematopodidae & Haematopus & $\begin{array}{l}\text { Haematopus } \\
\text { leucopodus }\end{array}$ & Pilpilén austral & & \\
\hline Charadriiformes & Haematopodidae & Haematopus & Haematopus palliatus & Pilpilén & & \\
\hline Anseriformes & Anatidae & Heteronetta & Heteronetta atricapilla & Pato rinconero & $\mathrm{LC}$ & \\
\hline Charadriiformes & Recurvirostridae & Himantopus & Himantopus mexicanus & Perrito & & \\
\hline Passeriformes & Hirundinidae & Hirundo & Hirundo rustica & Golondrina bermeja & & \\
\hline Passeriformes & Tyrannidae & Hymenops & Hymenops perspicillatus & Run-run & & \\
\hline Pelecaniformes & Ardeidae & Ixobrychus & Ixobrychus involucris & Huairavillo & LC & \\
\hline Charadriiformes & Laridae & Larosterna & Larosterna inca & Gaviotín monja & VU & \\
\hline Charadriiformes & Laridae & Larus & Larus dominicanus & Gaviota dominicana & & \\
\hline Gruiformes & Rallidae & Laterallus & Laterallus jamaicensis & Pidencito & NT & \\
\hline
\end{tabular}




\begin{tabular}{|c|c|c|c|c|c|c|}
\hline Orden & Familia & Género & Nombre científico & Nombre común & EC & $\mathrm{E}$ \\
\hline Passeriformes & Icteridae & Leistes & Leistes loyca & Loica & & \\
\hline Passeriformes & Furnariidae & Leptasthenura & $\begin{array}{l}\text { Leptasthenura } \\
\text { aegithaloides }\end{array}$ & Tijeral & & \\
\hline Passeriformes & Tyrannidae & Lessonia & Lessonia rufa & Colegial & & \\
\hline Charadriiformes & Laridae & Leucophaeus & Leucophaeus atricilla & Gaviota reidora & & \\
\hline Charadriiformes & Laridae & Leucophaeus & Leucophaeus modestus & Gaviota garuma & & \\
\hline Charadriiformes & Laridae & Leucophaeus & Leucophaeus pipixcan & Gaviota de Franklin & & \\
\hline Charadriiformes & Laridae & Leucophaeus & Leucophaeus scoresbii & Gaviota austral & & \\
\hline Charadriiformes & Scolopacidae & Limosa & Limosa haemastica & Zarapito de pico recto & & \\
\hline Anseriformes & Anatidae & Lophonetta & $\begin{array}{l}\text { Lophonetta } \\
\text { specularioides }\end{array}$ & Pato juarjual & & \\
\hline Procellariiformes & Procellariidae & Macronectes & Macronectes giganteus & Petrel gigante antártico & & \\
\hline Procellariiformes & Procellariidae & Macronectes & Macronectes halli & $\begin{array}{l}\text { Petrel gigante } \\
\text { subantártico }\end{array}$ & & \\
\hline Anseriformes & Anatidae & Mareca & Mareca sibilatrix & Pato real & & \\
\hline Coraciiformes & Alcedinidae & Megaceryle & Megaceryle torquata & Martín pescador & & \\
\hline Passeriformes & Thraupidae & Melanodera & $\begin{array}{l}\text { Melanodera } \\
\text { xanthogramma }\end{array}$ & Yal cordillerano & & \\
\hline Anseriformes & Anatidae & Merganetta & Merganetta armata & Pato cortacorrientes & NT & \\
\hline Columbiformes & Columbidae & Metriopelia & $\begin{array}{l}\text { Metriopelia } \\
\text { melanoptera }\end{array}$ & Tórtola cordillerana & & \\
\hline Falconiformes & Falconidae & Milvago & Milvago chimango & Tiuque & & \\
\hline Passeriformes & Mimidae & Mimus & Mimus patagonicus & Tenca patagónica & & \\
\hline Passeriformes & Mimidae & Mimus & Mimus thenca & Tenca & & \\
\hline Passeriformes & Mimidae & Mimus & Mimus triurus & Tenca de alas blancas & & \\
\hline Passeriformes & Icteridae & Molothrus & Molothrus bonariensis & Mirlo & & \\
\hline Passeriformes & Tyrannidae & Muscisaxicola & Muscisaxicola albilora & Dormilona de ceja blanca & & \\
\hline Passeriformes & Tyrannidae & Muscisaxicola & $\begin{array}{l}\text { Muscisaxicola } \\
\text { maclovianus }\end{array}$ & Dormilona tontita & & \\
\hline Passeriformes & Tyrannidae & Muscisaxicola & $\begin{array}{l}\text { Muscisaxicola } \\
\text { maculirostris }\end{array}$ & Dormilona chica & & \\
\hline Psittaciformes & Psittacidae & Myiopsitta & Myiopsitta monachus & Cotorra argentina & & \\
\hline Anseriformes & Anatidae & Netta & Netta peposaca & Pato negro & & \\
\hline Tinamiformes & Tinamidae & Nothoprocta & Nothoprocta perdicaria & Perdiz & & * \\
\hline Charadriiformes & Scolopacidae & Numenius & Numenius phaeopus & Zarapito & & \\
\hline Pelecaniformes & Ardeidae & Nycticorax & Nycticorax nycticorax & Huairavo & & \\
\hline Charadriiformes & Rostratulidae & Nycticryphes & $\begin{array}{l}\text { Nycticryphes } \\
\text { semicollaris }\end{array}$ & Becacina pintada & EN & \\
\hline Procellariiformes & Oceanitidae & Oceanites & Oceanites oceanicus & Golondrina de mar & & \\
\hline Charadriiformes & Charadriidae & Oreopholus & Oreopholus ruficollis & Chorlo de campo & & \\
\hline Caprimulgiformes & Trochilidae & Oreotrochilus & $\begin{array}{l}\text { Oreotrochilus } \\
\text { leucopleurus }\end{array}$ & Picaflor cordillerano & & \\
\hline Anseriformes & Anatidae & Oxyura & Oxyura ferruginea & Pato rana de pico ancho & & \\
\hline
\end{tabular}




\begin{tabular}{|c|c|c|c|c|c|c|}
\hline Orden & Familia & Género & Nombre científico & Nombre común & EC & E \\
\hline Anseriformes & Anatidae & Oxyura & Oxyura vittata & Pato rana de pico delgado & & \\
\hline Procellariiformes & Procellariidae & Pachyptila & Pachyptila belcheri & $\begin{array}{l}\text { Petrel - paloma de pico } \\
\text { delgado }\end{array}$ & & \\
\hline Procellariiformes & Procellariidae & Pachyptila & Pachyptila desolata & Petrel - paloma antártico & & \\
\hline Procellariiformes & Procellariidae & Pachyptila & Pachyptila vittata & $\begin{array}{l}\text { Petrel - paloma de pico } \\
\text { ancho }\end{array}$ & & \\
\hline Accipitriformes & Pandionidae & Pandion & Pandion haliaetus & Aguila pescadora & LC & \\
\hline Accipitriformes & Accipitridae & Parabuteo & Parabuteo unicinctus & Peuco & & \\
\hline Gruiformes & Rallidae & Pardirallus & $\begin{array}{l}\text { Pardirallus } \\
\text { sanguinolentus }\end{array}$ & Pidén & & \\
\hline Passeriformes & Passeridae & Passer & Passer domesticus & Gorrión & & \\
\hline Columbiformes & Columbidae & Patagioenas & Patagioenas araucana & Torcaza & LC & \\
\hline Caprimulgiformes & Trochilidae & Patagona & Patagona gigas & Picaflor gigante & & \\
\hline Procellariiformes & Procellariidae & Pelecanoides & Pelecanoides garnotii & Yunco & VU & \\
\hline Pelecaniformes & Pelecanidae & Pelecanus & Pelecanus thagus & Pelícano & NT & \\
\hline Suliformes & Phalacrocoracidae & Phalacrocorax & Phalacrocorax atriceps & Cormorán imperial & & \\
\hline Suliformes & Phalacrocoracidae & Phalacrocorax & $\begin{array}{l}\text { Phalacrocorax } \\
\text { bougainvillii }\end{array}$ & Guanay & VU & \\
\hline Suliformes & Phalacrocoracidae & Phalacrocorax & $\begin{array}{l}\text { Phalacrocorax } \\
\text { brasilianus }\end{array}$ & Yeco & & \\
\hline Suliformes & Phalacrocoracidae & Phalacrocorax & Phalacrocorax gaimardi & Lile & IC & \\
\hline Suliformes & Phalacrocoracidae & Phalacrocorax & $\begin{array}{l}\text { Phalacrocorax } \\
\text { magellanicus }\end{array}$ & Cormorán de las rocas & & \\
\hline Charadriiformes & Scolopacidae & Phalaropus & Phalaropus fulicarius & Pollito de mar rojizo & & \\
\hline Charadriiformes & Scolopacidae & Phalaropus & Phalaropus tricolor & Pollito de mar tricolor & & \\
\hline Falconiformes & Falconidae & Phalcoboenus & $\begin{array}{l}\text { Phalcoboenus } \\
\text { megalopterus }\end{array}$ & Carancho cordillerano & & \\
\hline Passeriformes & Furnariidae & Phleocryptes & Phleocryptes melanops & Trabajador & & \\
\hline Phoenicopteriformes & Phoenicopteridae & Phoenicopterus & $\begin{array}{l}\text { Phoenicopterus } \\
\text { chilensis }\end{array}$ & Flamenco chileno & NT & \\
\hline Passeriformes & Thraupidae & Phrygilus & Phrygilus alaudinus & Platero & & \\
\hline Passeriformes & Thraupidae & Phrygilus & Phrygilus fruticeti & Yal & & \\
\hline Passeriformes & Thraupidae & Phrygilus & Phrygilus gayi & Cometocino de Gay & & \\
\hline Passeriformes & Thraupidae & Phrygilus & Phrygilus patagonicus & Cometocino patagónico & & \\
\hline Passeriformes & Cotingidae & Phytotoma & Phytotoma rara & Rara & & \\
\hline Pelecaniformes & Threskiornithidae & Plegadis & Plegadis chihi & Cuervo de pantano & EN & \\
\hline Charadriiformes & Charadriidae & Pluvialis & Pluvialis squatarola & Chorlo ártico & & \\
\hline Podicipediformes & Podicipedidae & Podiceps & Podiceps major & Huala & & \\
\hline Podicipediformes & Podicipedidae & Podiceps & Podiceps occipitalis & Blanquillo & & \\
\hline Podicipediformes & Podicipedidae & Podilymbus & Podilymbus podiceps & Picurio & & \\
\hline Gruiformes & Rallidae & Porphyriops & Porphyriops melanops & Tagüita & & \\
\hline Procellariiformes & Procellariidae & Procellaria & $\begin{array}{l}\text { Procellaria } \\
\text { aequinoctialis }\end{array}$ & Fardela negra grande & & \\
\hline
\end{tabular}




\begin{tabular}{|c|c|c|c|c|c|c|}
\hline Orden & Familia & Género & Nombre científico & Nombre común & EC & E \\
\hline Procellariiformes & Procellariidae & Procellaria & Procellaria westlandica & Fardela de Nueva Zelanda & & \\
\hline Passeriformes & Hirundinidae & Progne & Progne chalybea & Golondrina doméstica & & \\
\hline Passeriformes & Furnariidae & Pseudasthenes & $\begin{array}{l}\text { Pseudasthenes } \\
\text { humicola }\end{array}$ & Canastero & & * \\
\hline Passeriformes & Tyrannidae & Pseudocolopteryx & $\begin{array}{l}\text { Pseudocolopteryx } \\
\text { citreola }\end{array}$ & Pájaro amarillo & & \\
\hline Procellariiformes & Procellariidae & Pterodroma & Pterodroma externa & $\begin{array}{l}\text { Fardela blanca de Juan } \\
\text { Fernández }\end{array}$ & EN & \\
\hline Passeriformes & Rhinocryptidae & Pteroptochos & Pteroptochos castaneus & Hued-hued castaño & & * \\
\hline Passeriformes & Rhinocryptidae & Pteroptochos & Pteroptochos tarnii & Hued-hued del Sur & & \\
\hline Passeriformes & Furnariidae & Pygarrhichas & Pygarrhichas albogularis & Comesebo grande & & \\
\hline Passeriformes & Hirundinidae & Pygochelidon & $\begin{array}{l}\text { Pygochelidon } \\
\text { cyanoleuca }\end{array}$ & $\begin{array}{l}\text { Golondrina de dorso } \\
\text { negro }\end{array}$ & & \\
\hline Podicipediformes & Podicipedidae & Rollandia & Rollandia rolland & Pimpollo & & \\
\hline Charadriiformes & Laridae & Rynchops & Rynchops niger & Rayador & & \\
\hline Passeriformes & Rhinocryptidae & Scelorchilus & Scelorchilus rubecula & Chucao & & \\
\hline Passeriformes & Rhinocryptidae & Scytalopus & Scytalopus fuscus & Churrín del norte & & * \\
\hline Passeriformes & Rhinocryptidae & Scytalopus & Scytalopus magellanicus & Churrín del Sur & & \\
\hline Caprimulgiformes & Trochilidae & Sephanoides & $\begin{array}{l}\text { Sephanoides } \\
\text { sephaniodes }\end{array}$ & Picaflor & & \\
\hline Passeriformes & Thraupidae & Sicalis & Sicalis auriventris & Chirihue dorado & & \\
\hline Passeriformes & Thraupidae & Sicalis & Sicalis flaveola & Chirihue azafrán & & \\
\hline Passeriformes & Thraupidae & Sicalis & Sicalis luteola & Chirihue & & \\
\hline Anseriformes & Anatidae & Spatula & Spatula cyanoptera & Pato colorado & & \\
\hline Anseriformes & Anatidae & Spatula & Spatula platalea & Pato cuchara & LC & \\
\hline Anseriformes & Anatidae & Spatula & Spatula versicolor & Pato capuchino & & \\
\hline Anseriformes & Anatidae & Speculanas & Speculanas specularis & Pato anteojillo & NT & \\
\hline Sphenisciformes & Spheniscidae & Spheniscus & Spheniscus humboldti & Pingüino de Humboldt & VU & \\
\hline Sphenisciformes & Spheniscidae & Spheniscus & $\begin{array}{l}\text { Spheniscus } \\
\text { magellanicus }\end{array}$ & Pingüino de Magallanes & & \\
\hline Passeriformes & Fringillidae & Spinus & Spinus barbatus & Jilguero común & & \\
\hline Passeriformes & Fringillidae & Spinus & Spinus uropygialis & Jilguero cordillerano & & \\
\hline Charadriiformes & Stercorariidae & Stercorarius & Stercorarius chilensis & Salteador chileno & & \\
\hline Charadriiformes & Stercorariidae & Stercorarius & Stercorarius longicaudus & Sallteador de cola larga & & \\
\hline Charadriiformes & Stercorariidae & Stercorarius & Stercorarius parasiticus & Salteador chico & & \\
\hline Charadriiformes & Laridae & Sterna & Sterna hirundinacea & Gaviotín sudamericano & & \\
\hline Charadriiformes & Laridae & Sterna & Sterna hirundo & Gaviotín boreal & & \\
\hline Charadriiformes & Laridae & Sterna & Sterna paradisaea & Gaviotín ártico & & \\
\hline Charadriiformes & Laridae & Sterna & Sterna trudeaui & Gaviotín piquerito & & \\
\hline Strigiformes & Strigidae & Strix & Strix rufipes & Concón & NT & \\
\hline Suliformes & Sulidae & Sula & Sula variegata & Piquero & IC & \\
\hline
\end{tabular}




\begin{tabular}{|c|c|c|c|c|c|c|}
\hline Orden & Familia & Género & Nombre científico & Nombre común & EC & $\mathrm{E}$ \\
\hline Passeriformes & Furnariidae & Sylviorthorhynchus & $\begin{array}{l}\text { Sylviorthorhynchus } \\
\text { desmursii }\end{array}$ & Colilarga & & \\
\hline Caprimulgiformes & Caprimulgidae & Systellura & Systellura longirostris & Gallina ciega & & \\
\hline Passeriformes & Tyrannidae & Tachuris & Tachuris rubrigastra & Sietecolores & & \\
\hline Passeriformes & Hirundinidae & Tachycineta & Tachycineta leucopyga & Golondrina chilena & & \\
\hline Procellariiformes & Diomedeidae & Thalassarche & Thalassarche bulleri & Albatros de Buller & & \\
\hline Procellariiformes & Diomedeidae & Thalassarche & $\begin{array}{l}\text { Thalassarche } \\
\text { melanophris }\end{array}$ & Albatros de ceja negra & $\mathrm{LC}$ & \\
\hline Procellariiformes & Diomedeidae & Thalassarche & Thalassarche salvini & Albatros de frente blanca & VU & \\
\hline Charadriiformes & Laridae & Thalasseus & Thalasseus elegans & Gaviotín elegante & & \\
\hline Pelecaniformes & Threskiornithidae & Theristicus & Theristicus melanopis & Bandurria & $\mathrm{LC}$ & \\
\hline Charadriiformes & Thinocoridae & Thinocorus & Thinocorus rumicivorus & Perdicita & & \\
\hline Charadriiformes & Scolopacidae & Tringa & Tringa flavipes & Pitotoy chico & & \\
\hline Charadriiformes & Scolopacidae & Tringa & Tringa melanoleuca & Pitotoy grande & & \\
\hline Charadriiformes & Scolopacidae & Tringa & Tringa semipalmata & Playero grande & & \\
\hline Passeriformes & Troglodytidae & Troglodytes & Troglodytes aedon & Chercán & & \\
\hline Passeriformes & Turdidae & Turdus & Turdus falcklandii & Zorzal & & \\
\hline Passeriformes & Tyrannidae & Tyrannus & Tyrannus savana & Cazamoscas tijereta & & \\
\hline Strigiformes & Tytonidae & Tyto & Tyto alba & Lechuza & & \\
\hline Passeriformes & Furnariidae & Upucerthia & Upucerthia dumetaria & Bandurrilla & & \\
\hline Passeriformes & Furnariidae & Upucerthia & Upucerthia saturatior & Bandurrilla de los bosques & & \\
\hline Charadriiformes & Charadriidae & Vanellus & Vanellus chilensis & Queltehue & & \\
\hline Cathartiformes & Cathartidae & Vultur & Vultur gryphus & Cóndor & NT & \\
\hline Charadriiformes & Laridae & Xema & Xema sabini & Gaviota de Sabine & & \\
\hline Passeriformes & Tyrannidae & Xolmis & Xolmis pyrope & Diucón & & \\
\hline Columbiformes & Columbidae & Zenaida & Zenaida auriculata & Tórtola & & \\
\hline Passeriformes & Passerellidae & Zonotrichia & Zonotrichia capensis & Chincol & & \\
\hline
\end{tabular}

